\title{
Das Recht als Archiv sozialer Konstruktionen der Migration
}

\author{
Jürgen Bast*
}
A. Einleitung .......................... 139
B. Die Gefahrenabwehr-Perspektive..... 141
I. Soziale Konstruktion: Migranten als Ordnungsproblem ................. 141
II. Charakteristische Bauform: die Aus- weisung ....................... 142
III. Anliegen der Migrationssteuerung: Abwehr und Überwachung gefährli- cher Personen..................... 144
C. Die zwischenstaatliche Perspektive.... 146
I. Soziale Konstruktion: Migranten als Staatsfremde ...................... 146
II. Charakteristische Bauform: das Nie- derlassungsabkommen ............. 146
III. Anliegen der Migrationssteuerung: Verfolgung von außenpolitischen Interessen ......................... 149
D. Die ökonomische Perspektive ........ 151
I. Soziale Konstruktion: Migranten als Humanressource ................... 151
II. Charakteristische Bauform: die Beschäftigungserlaubnis . 151

III. Anliegen der Migrationssteuerung: Wohlstandsmehrung und Schutz des inländischen Arbeitsmarktregimes . . 154

E. Die kulturelle Perspektive ............ 156

I. Soziale Konstruktion: Migranten als die Anderen...................... 156

II. Charakteristische Bauform: der Sprachtest..................... 157

III. Anliegen der Migrationssteuerung: Achtung kultureller Identitäten und Wahrung der „kulturellen Kompromisse“................................. 160

F. Die individualrechtliche Perspektive... 162 I. Soziale Konstruktion: Migranten als Rechtssubjekte................... 162

II. Charakteristische Bauform: das Refoulementverbot............... 162

III. Anliegen der Migrationssteuerung: Ermächtigung von Migranten ....... 166

G. Kopplung der Perspektiven als Aufgabe des Migrationsrechts ................. 169

\section{A. Einleitung}

Migration ist ein schillerndes Phänomen, in dessen Facetten sich höchst unterschiedliche Wertungen spiegeln, rechtliche wie politische. Man kann Migrationsprozesse vor allem als ein Problem der internationalen Sicherheit ansehen, oder aber primär als einen wirtschaftlichen Vorgang, oder als ein Menschenrechtsthema, um nur einige Interpretationen zu nennen. ${ }^{1}$ Keine dieser Wahrnehmungen ist richtig oder falsch; es handelt sich um normativ geprägte Aussagen, die einem sozialen Sachverhalt Bedeutungen zuschreiben und ihn dabei aus unterschiedlichen Blickwinkeln betrachten, ihn unterschiedlich „konstruieren“. Die Konstruktionsabhängigkeit und Bedeutungsvielfalt des Phänomens Migration kennzeichnet auch die Versuche, mit den Mitteln des Rechts lenkend in das Migrationsgeschehen einzugreifen.

Dieser Beitrag will die unterschiedlichen sozialen Konstruktionen identifizieren, die ihren Niederschlag im Recht der Migrationssteuerung gefunden haben. Dies geschieht in der Erwartung, dass den konkurrierenden Perspektiven auf Migranten und Migration charakteristische Rechtsinstitute und Regelungstechniken („Bauformen“)

* PD Dr. jur., Dipl.-Soz., Referent am Max-Planck-Institut für ausländisches öffentliches Recht und Völkerrecht, Heidelberg. Der Beitrag beruht auf der Habiliationsschrift des Verf., die unter dem Titel „Aufenthaltsrecht und Migrationssteuerung “ 2011 bei Mohr Siebeck erschienen ist.

1 Nach W. Walters/J. H. Haahr, Governing Europe, 2005, S. 98. 
entsprechen. ${ }^{2}$ Zwecks erkenntnisstiftender Verfremdung im diachronen Rechtsvergleich sollen hierzu Forschungsergebnisse der Neueren Rechtsgeschichte genutzt werden. ${ }^{3}$ Das Recht wird dabei als Teil des kulturellen Gedächtnisses verstanden, als ein Archiv sedimentierter Ideen, Erfahrungen und sozialer Beziehungen, auf das auch ein selektiver, von den Erkenntnisinteressen der Gegenwart bestimmter Zugriff möglich ist. ${ }^{4}$

Die folgende Untersuchung kombiniert Rechts- und Sozialwissenschaft. In ihren sozialtheoretischen Prämissen bezieht sie sich auf die in der Soziologie abweichenden Verhaltens ausformulierten Interaktionstheorien, die sich mit dem Namen Howard S. Becker verbinden. ${ }^{5}$ Dieser sog. Labeling Approach (Etikettierungstheorie) untersucht den Zusammenhang von Devianz und sozialer Kontrolle, also den gesellschaftlichen Prozess, in dem eine bestimmte Handlung oder Person als „abweichend“ definiert wird. ${ }^{6}$ Etikettierungstheorien wurden bisher nur selten auf die hoheitliche Reaktion auf Migrationsprozesse angewendet, und zudem lediglich auf bestimmte Einzelphänomene. Beispiele sind die Erforschung der polizeilichen Bekämpfung von „Zigeunern"7 und der frühneuzeitlichen Räuberbanden, die sich teilweise aus sozialen Gruppen mit ambulanten Lebensformen rekrutierten. ${ }^{8}$ Einer schlichten Übertragung des begrifflichen Rüstzeugs des Labeling Approach steht ohnehin entgegen, dass Migration nicht per se als abweichendes Verhalten etikettiert wird; Migrationsbiographien sind nicht stets Outsider-Karrieren, für die die Begriffe Abstemplung und Stigmatisierung einschlägig wären. Allerdings muss der Etikettierungsansatz von seinen Prämissen her nicht enggeführt werden auf eine Stigmatisierungs-Theorie. ${ }^{9}$ Angewendet auf die hoheitliche Steuerung der Migration heißt dies, die behördlichen Zuordnungen zu bestimmten Migranten-Kategorien als soziale Reaktion auf ein bestimmtes Verhalten zu begreifen, dem sie Ordnungsbegriffe „anheften“. Diese Etikettierung „von oben“ ist insofern maßgeblich, als sie die rechtlichen Konsequenzen determiniert, die sich gegen andere Selbstdefinitionen „von unten“ regelmäßig

2 Begriff nach E. Schmidt-Aßmann, Das allgemeine Verwaltungsrecht als Ordnungsidee, 2006, z.B. $3 / 152 \mathrm{ff}$.

3 Der zeitliche Schwerpunkt liegt auf der zweiten Hälfte des 19. Jahrhunderts, retrospektiv betrachtet die Phase der Entstehung des modernen Aufenthaltsrechts, siehe J. Torpey, The Invention of the Passport, 2000, S. 111-121; L. Lucassen, The Great War and the Origins of Migration Control in Western Europe and the United States, in: A. Böcker u.a. (Hrsg.), Regulation of Migration, 1998, S. 45-72; K. J. Bade, Europa in Bewegung, 2000, S. $232 \mathrm{f}$.

4 Zur Genealogie als Methode in der Tradition Foucaults siehe W. Walters, Mapping Schengenland, Environment and Planning D 20 (2002), S. 561, 562.

5 H. S. Becker, Außenseiter: Zur Soziologie abweichenden Verhaltens, 1973, S. 159-188; ferner etwa E. M. Lemert, Social Pathology, 1951.

6 Grundl. für die Rezeption in Deutschland F. Sack, Neue Perspektiven der Kriminalsoziologie, in: ders./ R. König, Kriminalsoziologie, 1968, S. 431-475; ferner etwa K. Lüderssen/F. Sack (Hrsg.), Abweichendes Verhalten I, 1975.

7 L. Lucassen, Zigeuner: Die Geschichte eines polizeilichen Ordnungsbegriffes, 1996.

8 U. Danker, Räuberbanden im Alten Reich um 1700, 1988.

9 H. Steinert, Zur Aktualität der Etikettierungs-Theorie, KrimJ 17 (1985), S. 29, 39 f. 
durchsetzen. ${ }^{10}$ Der soziologische Interaktionismus, wie ihn Becker für das Phänomen abweichenden Verhaltens entwickelt hat, liefert mithin eine taugliche theoretische Grundlage, das soziale Phänomen Migration von seinen variablen hoheitlichen Konstruktionen (= Perspektiven) her zu bestimmen.

Nicht zuletzt könnte die Einsicht in die Perspektivenpluralität dabei helfen, ein Denken in Dichotomien zu überwinden, die die rechtswissenschaftliche Behandlung von Migrationsfragen oftmals auszeichnet. Es hat eine lange Tradition, Fragen des Aufenthalts von Fremden auf dem eigenen Territorium aus dem Widerstreit von Partikularismus und Universalismus zu deuten: rechtsphilosophisch als Streit zwischen Kommunitarismus und Liberalismus, staatstheoretisch als Konflikt von territorialer Souveränität und universellen Menschenrechten, politisch als Kampf um restriktive oder liberale Zugangspolitiken, rechtsdogmatisch als Abwägung von öffentlichen Interessen an der Migrationssteuerung gegen private Migrationsinteressen. ${ }^{11}$ Die folgenden Überlegungen wollen dazu beitragen, die Statik dieses Arrangements zu überwinden.

\section{B. Die Gefahrenabwehr-Perspektive}

\section{Soziale Konstruktion: Migranten als Ordnungsproblem}

Die erste hier anzuführende Perspektive ist die Wahrnehmung von Migranten als eine potenzielle Bedrohung für die öffentliche Sicherheit und Ordnung, als ein polizeilich zu lösendes Ordnungsproblem. Diese soziale Konstruktion stand an der Wiege der Entstehung des Aufenthaltsrechts als „Fremdenpolizei“ und ist für seine rechtlichen Strukturen bis heute vielfach prägend. ${ }^{12}$

Die fremdenpolizeilichen Aktivitäten des 19. Jahrhunderts kreisten um drei Gruppen von Ordnungsproblemen, die von „gefährlichen Ausländern“ ausgehen: Armut, Kriminalität und Aufruhr. ${ }^{13}$ Migration wurde als Nährboden für „asoziale, kriminelle und politisch bedenkliche Elemente" 14 beargwöhnt, wobei fließende Übergänge bestanden zwischen den verschiedenen Gefahren für die öffentliche Ordnung, die mit Migranten assoziiert wurden, namentlich bei der Stigmatisierung sozialer Rand-

10 Ähnlich L. Lucassen, Zigeuner, 1996, S. 7-13.

11 Siehe nur J. Isensee, Die staatsrechtliche Stellung der Ausländer in der Bundesrepublik Deutschland, VVDStRL 32 (1974), S. 49, 50 f.; H. Hofmann, Die versprochene Menschenwürde, AöR 118 (1993), S. 353, 365-373; D. Thym, Migrationsverwaltungsrecht, 2010, S. 67 et passim; W. Kluth, Migrationsgerechtigkeit, ZAR 2011, S. 329-336; krit. L. Bosniak, The Citizen and the Alien, 2006, S. 4-11.

12 Rechtsvergl. M. Schmid-Drüner, Der Begriff der öffentlichen Sicherheit und Ordnung im Einwanderungsrecht ausgewählter EU-Mitgliedstaaten, 2007, S. 361-377.

13 F. Caestecker, The Transformation of Nineteenth-Century West European Expulsion Policy, in: A. Fahrmeir u.a. (Hrsg.), Migration Control in the North Atlantic World, 2003, S. 120, 121-123.

14 Zit. nach K. Schönwälder, Einwanderung und ethnische Pluralität, 2001, S. 217. 
gruppen und umherziehender Pauper als „Vagabunden" oder „Zigeuner"15 und bei der Abwehr von jüdischen Zuwanderern. ${ }^{16}$ Die Abgrenzung von Armuts- und Kriminalitätsbekämpfung war auch deshalb nicht trennscharf, weil fremdenpolizeilich relevante Erwerbsformen wie Prostitution oder Betteln vielerorts als Straftaten gewertet wurden. Zudem wuchs mit der Verschärfung der sozialen Konflikte im Zuge von Industrialisierung und Urbanisierung die Sorge vor politischen Unruhen, und mit ihr der fremdenpolizeiliche Kampf gegen vermeintliche Anarchisten und Sozialdemokraten sowie allgemein gegen „feindliche Ausländer“ und „Spione“, die aufgrund ihrer Nationalität als politisch illoyal eingestuft wurden. ${ }^{17}$

Sind Migranten einmal als latentes Ordnungsproblem definiert, legitimiert sich die Entwicklung von Techniken der systematischen Erfassung und Überwachung: Es wird eine gesonderte ausländerrechtliche „Disziplinarordnung“ für sie aufgestellt. ${ }^{18}$ Ein von der Gefahrenabwehr-Perspektive geprägtes Aufenthaltsrecht konstituiert mithin eine zweite Schicht der staatlichen Kontrolle abweichenden Verhaltens, zusätzlich zu den für alle Einwohner geltenden Verhaltensnormen und Sanktionsdrohungen des allgemeinen Polizeirechts bzw. Strafrechts.

\section{Charakteristische Bauform: die Ausweisung}

Die Aufgabe der präventiven Kontrolle abweichenden Verhaltens der Ausländerbevölkerung durch besondere Ordnungsbehörden geht einher mit einem spezifischen Eingriffs- und Sanktionsinstrumentarium. Dies hat zur Herausbildung zahlreicher Rechtsinstitute der ausländerpolizeilichen Überwachung geführt, etwa besondere Ausweis- und Meldepflichten oder Beschränkungen für die politische Betätigung. $\mathrm{Zu}$ nennen sind hier auch polizeitechnische Innovationen wie die Ausländerakte oder zentrale Datensammlungen, die der Koordinierung der ausländerbehördlichen Aktivitäten dienen. Ihre spezifisch aufenthaltsrechtliche Prägung erhalten diese Bauformen aber erst durch das Sanktionsinstrument, um das sie gruppiert sind: die mit Zwangsmitteln durchsetzbare Aufenthaltsbeendigung. Das unbestrittene Zentralinstitut der Gefahrenabwehr-Perspektive auf das Migrationsgeschehen ist die Ausweisung mit ihren vollstreckungsrechtlichen Hilfsinstituten, allen voran die Abschiebung und die Schubhaft.

15 Ausf. L. Lucassen, Zigeuner, 1996, zusf. S. 214-230; M. Zimmermann, Ausgrenzung, Ermordung, Ausgrenzung, in: A. Lüdtke (Hrsg.), „Sicherheit“ und „Wohlfahrt“, 1992, S. 344-370.

16 T. van Rahden, Die Grenze vor Ort, Tel Aviver Jahrbuch für deutsche Geschichte 27 (1998), S. 47, 64-69; D. Gosewinkel, „Unerwünschte Elemente“, ebd., S. 71, 90-104; A. R. Zolberg, Global Movements, Global Walls, in: Wang Gungwu (Hrsg.), Global History and Migrations, 1997, S. 279, 300303.

17 Exempl. I. Reiter, Ausgewiesen, abgeschoben, 2000, S. 387-420; H. Motomura, Americans in Waiting, 2006, S. 38-45.

18 Begriff nach H. H. Heldmann, Ausländerrecht: Disziplinarordnung für die Minderheit, 1974. 
Die besondere Nähe des Rechtsinstituts zur Perspektive der Gefahrenabwehr ist Teil seiner Geschichte. Nach der Reichsgründung existierten in Deutschland verschiedene Formen der Ausweisung nebeneinander. ${ }^{19}$ Einerseits gab es die Reichsverweisung, die im Anschluss an ein Strafurteil vorgesehen, in der Praxis aber selten war, andererseits die landesrechtliche Ausweisung in Gestalt eines gesetzlich ungebundenen und gerichtlich nicht kontrollierten Ausweisungsermessens der Polizeibehörden. ${ }^{20}$ Für eine polizeiliche Landesverweisung genügten jegliche Gründe der öffentlichen Wohlfahrt. Ansässige Ausländer konnten mithin Adressaten von Maßnahmen der Fremdenpolizei werden, ohne dass ihr Verhalten eine Gefahr im Sinne des liberalen Polizeirechts des Kreuzberg-Urteils vom 14. Juni $1882^{21}$ begründen musste.

Unter der Weimarer Verfassung wurde die Schaffung einer gesetzlichen Grundlage für die Ausweisung jenseits der landespolizeilichen Generalklauseln diskutiert, ohne sich jedoch durchsetzen zu können. ${ }^{22}$ Die überwiegende Ansicht ging weiterhin von einem freien Ausweisungsermessen auch bei bloßer wirtschaftlicher oder sonstiger „Lästigkeit“ aus, also unterhalb der Schwelle einer polizeirechtlichen Gefahr. ${ }^{23}$ Bis 1938 bestand gar keine reichsrechtliche Regelung der polizeilichen Ausweisung, bis 1965 keine parlamentarisch-gesetzliche. Sowohl in der Ausländerpolizeiverordnung (APVO) des RMI von 1938, die unter dem Grundgesetz als Rechtsgrundlage für Ausweisungen zunächst fortgalt, als auch nach dem AuslG 1965 bliebt es bei der Regelung, dass eine individuell zurechenbare Gefahr für die öffentlichen Sicherheit oder Ordnung von dem Ausgewiesenen nicht ausgehen muss. Auf die anders deutbare Formulierung der APVO 1938, die auf das „Verhalten“ des Ausländers abstellte und aufgrund der Rechtsschutzgarantie des Grundgesetzes auch justiziabel geworden war, hat der Gesetzgeber des AuslG 1965 verzichtet. Verlangt war nur noch, dass „seine Anwesenheit erhebliche Belange der Bundesrepublik Deutschland ... beeinträchtigt“ ( $\$ 10$ Abs. 1 Nr.11 AuslG 1965). Diese „formelle Entpolizeilichung des Aufenthaltsrechts " 24 diente ersichtlich der Abschirmung eines weiten Ausweisungsermessens gegen mögliche Interferenzen durch die Gerichte.

Diese blieben gleichwohl letztlich nicht aus. Infolge des grundgesetzlichen Verhältnismäßigkeitsprinzips und vor allem unter dem Einfluss der EMRK hat das Auswei-

19 Im Überblick A. Bordewin, Das Aufenthaltsrecht der Ausländer, 1962, S. 8-14; H. H. Friederichsen, Die Stellung des Fremden, 1967, S. 80-84; G. Renner, Ausländerrecht in Deutschland, 1998, $\mathbb{S} 4$ Rn. 21-31.

20 Vgl. $\$ 130$ Abs. 3 des preußischen Gesetzes über die allgemeine Landesverwaltung vom 30. Juli 1883, PrGS 1883, 195, wonach verwaltungsgerichtlicher Rechtsschutz nicht zugelassen war; ebenso $\int \mathbb{S} 41$ ff. der preußischen Polizeiverordnung über die Behandlung von Ausländern vom 27. April 1932, PrGS 1932, 179.

21 OVGE 9, 353-384.

22 J. Ziekow, Über Freizügigkeit und Aufenthalt, 1997, S. 308.

23 W. Kobarg, Ausweisung und Abweisung von Ausländern, 1930, S.66-69, E. Isay, Das deutsche Fremdenrecht, 1923, S. 97-100, 226-227.

24 K. Dohse, Ausländische Arbeiter und bürgerlicher Staat, 1981, S. 247. 
sungsrecht massive Veränderungen erfahren..$^{25}$ Gegen die dominante Konstruktion von Migranten als Ordnungsproblem hat sich die - noch näher zu erläuternde individualrechtliche Perspektive zunehmend Beachtung und rechtliche Relevanz im Ausweisungsrecht verschafft. Die Kontinuitätslinien zur Fremdenpolizei des 19. Jahrhunderts sind im Text des geltenden AufenthG dennoch weiterhin unschwer zu erkennen, etwa wenn Straffälligkeit, Obdachlosigkeit und Sozialhilfebezug als reguläre Ausweisungsgründe normiert sind und jeder für sich im Regelfall die Versagung eines Aufenthaltstitels begründet. Wenn für das heutige Migrationsrecht generell ein Trend zur Konstruktion von Migration als Sicherheitsproblem („securitization of migration") beobachtet ${ }^{26}$ und die Dominanz einer polizeilichen Sicht auf Migranten konstatiert wird, die durch die Nutzung des Aufenthaltsrechts für Zwecke der Terrorismusbekämpfung noch verstärkt wurde, ${ }^{27}$ spricht dies für eine Renaissance der Gefahrenabwehr-Perspektive. Für den deutschen Kontext ist jedoch zugleich festzuhalten, dass für die Vorherrschaft dieser Perspektive speziell im Ausweisungsrecht nicht zuletzt die frühe Abkopplung von den Entwicklungen hin zu einem rechtsstaatlichen Polizeirecht verantwortlich war.

\section{Anliegen der Migrationssteuerung: Abwehr und Überwachung gefährlicher Personen}

Welchen speziellen Zugriff auf die Steuerung der Migration bringt die Gefahrenabwehr-Perspektive ein, gibt es ein übergreifendes Anliegen der Migrationssteuerung, das sie bündelt und artikuliert?

Hierzu gilt es zunächst darauf hinzuweisen, dass die Bemühungen um eine vorsorgende Steuerung der Migration mit den rechtlichen Instrumenten des Aufenthaltsrechts ein Phänomen des 20. Jahrhunderts sind. Der traditionelle Gegenstand der Ausländerpolizei war und ist nicht die Gestaltung von Migrationsprozessen, sondern die - tendenziell umfassend verstandene - Überwachung von ansässigen Ausländern. In der Indienstnahme der polizeilichen Eingriffs- und Sanktionsinstrumente für Zwecke einer an öffentlichen Interessen orientierten Zuwanderungspolitik liegt ein grundlegender Funktionswandel hin zu interventionsstaatlicher Wohlfahrtspflege. ${ }^{28}$

25 D. Thym, Migrationsverwaltungsrecht, 2010, S. 201-255; J. Bast, Aufenthaltsrecht und Migrationssteuerung, 2011, S. 180-182, 190-203.

26 J. Huysmans, The Politics of Insecurity, 2006, S. 45-62.

27 D. Bender, „Verpolizeilichung“ des Ausländerrechts?, KJ 2003, S. 130, 144.

28 F. Caestecker, The Transformation of Nineteenth-Century West European Expulsion Policy, in: A. Fahrmeir u.a. (Hrsg.), Migration Control in the North Atlantic World, 2003, S. 120, 130-132; zum Zusammenhang von „welfarism“ und „deportation“ und zur Deutung der Ausweisungs- und Abschiebepraxis als moderne Instrumente staatlicher Bevölkerungskontrolle siehe W. Walters, Deportation, Expulsion, and the International Police of Aliens, Citizenship Studies 6 (2002), S. 265, 279-284. 
Dieser Zusammenhang wird besonders deutlich, wenn man ihn mit dem umgekehrten Entwicklungsweg kontrastiert, den das US-Einwanderungsrecht gegangen ist. ${ }^{29}$ Die deportation erfüllte dort nach herkömmlichem Verständnis in erster Linie die Funktion, Lücken bei der Einwanderungskontrolle an der Grenze zu kompensieren („extended border control“). Erst später entwickelte sich die weitere Funktion der Einwandererkontrolle, einschließlich der Verknüpfung mit der Strafjustiz („postentry social control“). So war die Ausweisung von verarmten oder straffälligen Einwanderern zunächst nur innerhalb einer bestimmten Frist nach der Einreise möglich: ab 1891 für ein Jahr, ab 1917 fünf Jahre nach der Ankunft. Dem lag nicht etwa die Vorstellung einer mit zunehmender Aufenthaltsdauer wachsenden Bindung zum Aufenthaltsstaat zu Grunde; diese Idee von migration as affiliation passt eher zum europäischen Verständnis der Ausweisung. Vielmehr verblasst mit dem Zeitablauf die Verbindung zum Grenzübertritt, sodass eine „nachgelagerte Grenzkontrolle“ unplausibel wird.

Nach dem Bisherigen erscheint die Gefahrenabwehr-Perspektive als mehr oder weniger strikt auf eine restriktive Einwanderungspolitik verpflichtet. Sie würde daher nicht dem Bild einer für unterschiedliche politische Gestaltungen offenen Sichtweise entsprechen. Dies trifft jedenfalls insofern zu, als sie mit ihrer Fixierung auf migrationsbedingte Bedrohungslagen auf die Formulierung negativer Auswahlkriterien für unerwünschte Migranten festgelegt ist, auch wenn die Kriterien selbst inhaltlich variabel sind und unterschiedliche politische Haltungen reflektieren können. Man kann dies so verallgemeinern, dass eine Migrationssteuerung aus der GefahrenabwehrPerspektive das Anliegen verfolgt, diejenigen Migranten(-kategorien) zu identifizieren, von denen eine tatsächliche oder prognostizierte Beeinträchtigung öffentlicher Interessen ausgeht und deren (dauerhafte) Anwesenheit deshalb unerwünscht ist.

Deshalb ist es auf den ersten Blick überraschend, dass sich ein von dieser Perspektive beherrschtes Ausweisungsrecht in bestimmten historischen Perioden mit einer liberalen Einwanderungspolitik und einem freizügigen Einreise- und Niederlassungsregime verbunden hat. Auf den zweiten Blick beruht dies auf einer einleuchtenden Kompensationslogik: Ein weitgehend ungebundenes Ausweisungsermessen kann als Rückversicherung dienen, um auf präventive Einreisekontrollen ganz zu verzichten, ${ }^{30}$ oder doch zumindest eine liberale Handhabung der präventiven Kontrollinstrumente legitimieren, solange diese Liberalität auf Generosität und nicht auf Rechtsansprüchen der Migranten beruht. ${ }^{31}$

Es ist dieses paradigmatische Zusammenspiel von liberaler Zulassungs- und restriktiver Ausweisungspraxis, geformt unter den verfassungsrechtlichen und ideologi-

29 Zum Folgenden D. Kanstroom, Deportation Nation, 2007, S. 4-6, 91-130.

30 So das Modell des Passwesen-Gesetzes von 1867, das formell erst 1937 aufgehoben wurde.

31 So im Kern das deutsche Modell seit der Wiederanwendung der APVO 1938 unter dem GG. 
schen Prämissen der politisch autoritären und wirtschaftlich liberalen Monarchie, das durch die individualrechtliche Überformung des Ausweisungsrechts in Frage gestellt wird. Heute, unter dem Einfluss der EMRK, hat sich im europäischen Rechtsraum eine Einengung des staatlichen Ausweisungsermessens durchgesetzt, die mit einer verstärkten Berücksichtigung der privaten Interessen an der Fortsetzung des Aufenthalts einhergeht. Vor diesem konzeptionellen Hintergrund wird die Aufwertung der öffentlichen Interessen bei der Entscheidung über die (erstmalige) Zulassung, wie sie $\mathbb{} 1$ AufenthG verkündet, verständlich. Die privaten Migrationsinteressen rücken in dem Maße bei der Zuwanderung in den Hintergrund, wie sie in Gestalt von Bleiberechten im Ausweisungsrecht verstärkt berücksichtigt werden. Dies entlastet im Gegenzug das Ausweisungsrecht davon, der maßgebliche Hebel der Geltendmachung öffentlicher Interessen zu sein. Das Aufenthaltsrecht kann perspektivisch darauf umstellen, die öffentlichen Interessen, die sich nicht als Gefahrenabwehr im polizeirechtlichen Sinne darstellen, vorrangig in den Verfahren zur Genehmigung des Aufenthalts zu berücksichtigen.

\section{Die zwischenstaatliche Perspektive}

\section{Soziale Konstruktion: Migranten als Staatsfremde}

Die zweite soziale Konstruktion, die im Aufenthaltsrecht wirkungsmächtig wird, ist die Bezugnahme auf Migranten als Staatsfremde, als Angehörige einer anderen Staatsmacht, deren Anwesenheit auf dem eigenen Staatsgebiet einen Vorgang von zwischenstaatlicher Relevanz schafft. Der maßgebliche Anknüpfungspunkt ist die Staatsangehörigkeit des Migranten, die eine rechtliche Zuordnung zu einem anderen Staat, unter Umständen auch eine politisch-ideologische Zuordnung zu dessen Regierung beinhaltet und nach einer Zuständigkeitsabgrenzung zwischen den beteiligten Völkerrechtssubjekten verlangt. Im Hintergrund steht die durch den Migrationsvorgang aktualisierte Spannung zwischen Gebiets- und Personalhoheit, zwischen Territorialität und Mitgliedschaft: der Staatsfremde wird zum Objekt zweier Gewalten und tritt beiden als möglicher Träger von Rechten gegenüber. Aus der zwischenstaatlichen Perspektive begründet der Ausländeraufenthalt mithin ein dreipoliges Rechtsverhältnis. ${ }^{32}$

\section{Charakteristische Bauform: das Niederlassungsabkommen}

Auf der Suche nach einer Bauform, die die Eigenarten dieser Perspektive am besten zur Sprache bringt, stößt man zunächst auf das Institut der Passpflicht. Sie weist Bezüge zu allen drei Schenkeln des Fremdenrechtsverhältnisses auf. Auf der einen Seite manifestiert sich im Passzwang der umfassende Zugriff des modernen Staates

32 K. Doehring, Die allgemeinen Regeln des völkerrechtlichen Fremdenrechts, 1963, S. 54; J. Isensee, Die staatsrechtliche Stellung der Ausländer in der Bundesrepublik Deutschland, VVDStRL 32 (1974), S. 49, 55-57. 
auf grenzüberschreitende Migrationsvorgänge, also das Herrschaftsverhältnis zwischen Migrant und Aufenthaltsstaat. ${ }^{33}$ Auf der anderen Seite ist der „Pass“ nicht nur alltagssprachlich ein Synonym für Staatsangehörigkeit, er bringt die rechtliche Verbindung zwischen dem ausstellendem Staat und seinen Angehörigen zum Ausdruck. Die Ausstellung von Reisedokumenten an Ausländer ist im 19. Jahrhundert praktisch ausgestorben und wird heute nur noch in besonderen Konstellationen praktiziert, insbesondere bei Flüchtlingen. Die rechtliche Beziehung zwischen den beteiligen Staaten ist insofern berührt, als der Pass im zwischenstaatlichen Verhältnis als Primafacie-Beweis der Staatsangehörigkeit seines Inhabers dient. Seine Ausstellung durch die Behörden des Herkunftsstaats beinhaltet nicht nur eine Reiseerlaubnis, sondern auch die konkludente Bereitschaft zur Rückübernahme. ${ }^{34}$ Bei der Auferlegung einer Passpflicht für einreisende Ausländer geht es nicht zuletzt darum sicherzustellen, eine spätere Ausreisepflicht tatsächlich durchsetzen zu können, ausgehend von der im Grundsatz unbestrittenen völkerrechtlichen Pflicht zur Rückübernahme eigener Staatsangehöriger. ${ }^{35}$

Bedenken gegen die Inthronisierung des Passzwangs als zentrale Bauform der zwischenstaatlichen Perspektive können sich daran festmachen, dass der Passzwang historisch gesehen auch und vor allem polizeilichen Zwecken diente, insbesondere der Überwachung der Reisenden entlang der dokumentierten Reiseroute und am Aufenthaltsort. Der Historiker Andreas Fahrmeir hat detailliert nachvollzogen, wie sich zu Beginn des 19. Jahrhunderts in den deutschen Staaten das staatliche Passmonopol durchgesetzt hat. ${ }^{36}$ Der Pass wandelte sich von einer Art Empfehlungsschreiben, das den guten Leumund des Inhabers bezeugte, zu einer Reisegenehmigung des Heimatlandes, die zugleich als Identitätsdokument diente. Nach französischem Vorbild wurde die Passpflicht für Ausländer hauptsächlich zu polizeilichen Zwecken im Inland eingesetzt, wobei die Kriminalitätsbekämpfung und die Beobachtung sozialer Randgruppen im Vordergrund standen. In den Debatten der zweiten Hälfte des 19. Jahrhunderts über die Abschaffung bzw. Aussetzung der Passpflicht ging es nicht zuletzt darum, den allgegenwärtigen polizeilichen Zugriff auf die Reisenden abzumildern, der als nicht mehr zeitgemäß empfunden wurde. ${ }^{37}$ Genetisch gehört die Passpflicht daher zu den Instituten, die mit der Gefahrenabwehr-Perspektive zumindest ebenso verschwistert sind wie mit der zwischenstaatlichen.

33 J. Torpey, The Invention of the Passport, 2000, S. 4-20; L. Lucassen, A Many-Headed Monster: The Evolution of the Passport System, in: J. Caplan/J. Torpey (Hrsg.), Documenting Individual Identity, 2001, S. 235, 238-255.

34 G. S. Goodwin-Gill, International Law and the Movement of Persons between States, 1978, S. 44-46.

35 K. Hailbronner, Rückübernahme eigener und fremder Staatsangehöriger, 1996, S. 28-34.

36 A. Fahrmeir, Citizens and Aliens, 2000, S. 101-129; ders., Paßwesen und Staatsbildung in Deutschland des 19. Jahrhunderts, HZ 271 (2000), S. 57, 60-77.

37 J. Torpey, The Invention of the Passport, 2000, S. 79-82. 
Zum Charakter der zwischenstaatlichen Perspektive würde ohnehin eine Bauform aus dem Völkerrecht besser passen. In Frage kommt hier die aufenthaltsrechtliche Privilegierung aufgrund der Staatsangehörigkeit, deren klassische Form das Niederlassungsabkommen ist. Die Hochphase dieses Abkommenstypus war die zweite Hälfte des langen 19. Jahrhunderts, er stellt gewissermaßen die kooperative Entsprechung zur einseitigen Aufhebung von Visapflichten und Passkontrollen dar. ${ }^{38}$ Teilweise sind die typischen Klauseln von Niederlassungsverträgen eingebettet in einen sog. Freundschafts-, Handels- und Schifffahrtsvertrag, in dem weitere Fragen der bilateralen diplomatischen und wirtschaftlichen Beziehungen geregelt werden. ${ }^{39}$ In der Sache geht es um Regelungen zum Schutz und Vorteil der eigenen, im Ausland lebenden Staatsangehörigen, wobei infolge des Grundsatzes der Gegenseitigkeit gleichzeitig eine Privilegierung der im Staatsgebiet lebenden Angehörigen des Vertragspartners entsteht. ${ }^{40}$ Typische aufenthaltsrechtliche Bestimmungen sind die $\mathrm{Zu}$ sicherung, dass sich die Angehörigen des Vertragspartners „nach Maßgabe der Gesetze“ im eigenen Staatsgebiet frei aufhalten und niederlassen dürfen, sowie die $\mathrm{Zu}$ sicherung von Inländerbehandlung, Meistbegünstigung oder zumindest wohlwollender Prüfung bei der Zulassung zum (selbständigen) Gewerbe, je nach dem intendierten Grad der Privilegierung. Diese Freizügigkeit eröffnenden Regelungen stehen aber - ausdrücklich oder implizit - unter dem Vorbehalt fremdenpolizeilicher Maßnahmen, einschließlich der Ausweisung aus „Gründen der Armen- und Sittenpolizei““. ${ }^{41}$ Teilweise regeln die Niederlassungsverträge auch die Übernahmepflichten und -modalitäten im Fall der Abschiebung mit. ${ }^{42}$

Bei den Niederlassungsabkommen, wie bei der zwischenstaatlichen Perspektive insgesamt, dominieren Bauformen des Völkerrechts alter Prägung: einer Koordinationsordnung zur Regelung der intergouvernementalen Beziehungen und zur Beilegung zwischenstaatlicher Konflikte. Man trifft hier auf das völkergewohnheitsrechtliche Fremdenrecht, das sich mit den Mindestanforderungen an die Behandlung fremder Staatsangehöriger befasst, wobei deren Verletzung den Heimatstaat zur Ausübung diplomatischen Schutzes berechtigt. ${ }^{43}$ Die Niederlassungsverträge modifizieren diesen Standard durch partikuläres Fremdenrecht, indem sie auf der Basis von Gegenseitigkeit eine privilegierte Behandlung zusichern, ohne die koordinati-

38 Zum Bestand der Verträge des Deutschen Reichs siehe A. Heinrichs, Deutsche Niederlassungsverträge und Übernahmeabkommen, 1908, S. 17-139.

$39 \mathrm{Zu}$ diesem Vertragstypus siehe A. Paulus, Treaties of Friendship, Commerce and Navigation in: R. Wolfrum (Hrsg.), MP-EPIL online (Stand: März 2007).

40 O. Kimminich, in: Bonner Kommentar (20. Lfg. 1968), Art. 74 Nr. 4 GG, Rn. 15.

41 So Art. 4 des dt.-schweiz. Niederlassungsvertrags vom 31. Mai 1890 (RGBl. 1890, 131); näher A. von Overbeck, Niederlassungsfreiheit und Ausweisungsrecht, 1907, S. 86-89.

42 Etwa Art. 6-13 des dt.-niederl. Niederlassungsvertrags vom 17. Dezember 1904 (RGBl. 1906, 879).

43 G. Dahm/J. Delbrück/R. Wolfrum, Völkerrecht, Bd. I/2, 2. Aufl. 2002, S. 115-123, 130-137. 
onsrechtliche Struktur als solche zu verlassen. ${ }^{44}$ Erst eine jüngere Generation von Niederlassungsverträgen, die nach dem Zweiten Weltkrieg zwischen den europäischen Staaten geschlossen wurden, geht darüber partiell hinaus, indem sie Beschränkungen der staatlichen Ausweisungsbefugnisse etablieren und sich damit für eine individualrechtliche Perspektive öffnen. ${ }^{45}$ Ein wichtiger Schritt war in diesem Zusammenhang die Multilateralisierung der wechselseitigen Privilegierungen im Rahmen des Europarates durch das Europäisches Niederlassungsabkommen vom 13. Dezember $1955,{ }^{46}$ das einen besonderen Ausweisungsschutz für dauerhaft Niedergelassene beinhaltet. Noch weiter auf diesem Weg ging der EWG-Vertrag vom 1957, der ein mehrseitiges Niederlassungsabkommen ist, das die früheren bilateralen Verträge zwischen den sechs Gründungsstaaten praktisch überholte. ${ }^{47}$

\section{Anliegen der Migrationssteuerung: Verfolgung von außenpolitischen Interessen}

Bei der Suche nach einem spezifischen Anliegen, das bei der Migrationssteuerung aus einer zwischenstaatlichen Perspektive verfolgt wird, ist zunächst zu vergegenwärtigen, dass das völkerrechtliche Fremdenrecht allenfalls mittelbar zur Migrationssteuerung diente und für diesen Zweck letztlich auch wenig geeignet ist. Der begrenzte Bestand an materiellen Regeln und der Durchsetzungsmechanismus diplomatischen Schutzes bieten immerhin ein gewisses Maß an Rechtssicherheit für den grenzüberschreitenden Verkehr und dienen so den Migrationsinteressen der transnationalen Händler- und Geschäftswelt. ${ }^{48}$ Diesem mobilitätsfördernden, liberalen Impetus folgten auch die bilateralen Freundschafts- und Niederlassungsverträge des 19. Jahrhunderts, deren Regelungen zu Enteignungsschutz und Meistbegünstigung heute eher im internationalen Wirtschaftsrecht denn im migrationsrechtlichen Kontext verhandelt würden.

Auf das wirtschaftsliberale Leitbild ihres Entstehungszusammenhangs sind die entsprechenden völkerrechtlichen Bauformen aber nicht festgelegt. Auf einer allgemeineren Betrachtungsebene begründen die rechtliche Anknüpfung an und die Unterscheidung nach der Staatsangehörigkeit der Migranten, wie sie für die zwischenstaatliche Aushandlung aufenthaltsrechtlicher Fragen typisch sind, eine besondere Beziehung der zwischenstaatlichen Perspektive zur auswärtigen Politik. Gemeint sind damit nicht die Versuche, Migrationssteuerung mit den Mitteln der Außenpolitik zu betreiben. ${ }^{49}$ Angesprochen ist vielmehr die Verfolgung außenpolitischer Ziele mit

44 Zum zeitgenössischen Verständnis siehe H. von Frisch, Das Fremdenrecht, 1910, S. 129-136.

45 Z.B. Art. III des dt.-franz. Niederlassungs- und Schifffahrtsvertrag vom 27. Oktober 1956, BGBl. 1957 II 1661.

46 BGBl. 1959 II 997.

47 Vgl. U. Everling, Das Niederlassungsrecht im Gemeinsamen Markt, 1963, S. 22 f.

48 Vgl. C. Schmitt, Der Nomos der Erde im Völkerrecht des Jus Publicum Europaeum, 1950, S. 208-209.

49 R. Münz/M. Weiner, Conclusion, in: dies. (Hrsg.), Migrants, Refugees, and Foreign Policy, 1997, S. 323-355. 
aufenthaltsrechtlichen Mitteln, etwa durch gezielte Privilegierung der Angehörigen ausgewählter Vertragspartner. Als spezifischer Ansatz der Migrationssteuerung aus zwischenstaatlicher Perspektive kann mithin die Verfolgung von bzw. der Abgleich mit außenpolitischen Interessen ausgezeichnet werden.

Eine bestimmte migrationspolitische Ausrichtung ist damit nicht präjudiziert. Politikwissenschaftliche Studien haben komplexe Wechselbeziehungen aufgezeigt zwischen der konkreten Ausgestaltung des Aufenthaltsrechts und den internationalen Beziehungen zu den Herkunftsstaaten, ${ }^{50}$ aber auch zwischen außenpolitischem Handeln und internationalen Migrationsprozessen, zumal wenn es sich um Flüchtlingsbewegungen handelt. ${ }^{51}$ Außenpolitik und Migrationspolitik stellen in modernen Regierungssystemen funktionell verselbständigte Politikbereiche dar, die von unterschiedlichen staatlichen Stellen in relativer Autonomie bearbeitet werden. Normalerweise schlagen außenpolitische Interessen nicht unmittelbar auf den Vollzug aufenthaltsrechtlicher Bestimmungen durch. Vielmehr ist es eine der Aufgaben des Rechts, in spezifischen Konstellationen die Einbeziehung der zwischenstaatlichen Perspektive in migrationsbezogenen Entscheidungen zu ermöglichen, ohne außenpolitischen Zielen per se Vorrang einzuräumen. Kritische Punkte sind beispielsweise die Aufnahme von Flüchtlingsgruppen im Rahmen von Resettlement-Programmen oder die prioritäre Anwerbung von Arbeitskräften aus bestimmten Herkunftsländern. Hier werden Mechanismen benötigt, um die Kopplung der Perspektiven zu ermöglichen: zum Abgleich von außenpolitischem Realismus mit humanitären Verpflichtungen im ersten Fall, mit ökonomischen Interessen im zweiten Fall.

Was den Abschluss von Niederlassungsverträgen als historisch verbürgte Form aufenthaltsrechtlicher Privilegierung angeht, scheinen diese - zumindest für Deutschland ist dies sicher - eine abnehmende Bedeutung zu haben. ${ }^{52}$ Der disparat wirkende Bestand der heute noch operativen Verträge mit Nicht-EU-Staaten demonstriert, dass die Institutionalisierung von Privilegierungspolitiken in Form von bilateralen Verträgen das Risiko mangelnder Flexibilität birgt, wenn Alt-Verträge mittlerweile überholte außenpolitische Interessenlagen spiegeln. ${ }^{53}$ Vielleicht sind die Verwaltungsgerichte auch deshalb zurückhaltend, den Bestimmungen dieser als Fremdkörper im geltenden Aufenthaltsrecht empfundenen Verträge eine gehaltvolle Bedeutung

50 M. Weiner, On International Migration and International Relations, PDR 11 (1985), Nr. 3, S. 441, 447-452.

51 M. S. Teitelbaum, Immigration, Refugees, and Foreign Policy, IO 38 (1984), S. 429, 433-443; Ch. Mitchell, International Migration, International Relations and Foreign Policy, IMR 23 (1989), S. 681, 688-690; F. Nuscheler, Migration als Konfliktquelle und internationales Ordnungsproblem, in: P. Imbusch/R. Zoll (Hrsg.), Friedens- und Konfliktforschung, 2005, S. 275-285.

52 Vgl. dagegen frühere Einschätzungen bei R. B. Lillich, The Human Rights of Aliens in Contemporary International Law, 1984, S. $121 \mathrm{f}$.

53 J. Bast, Internationalisierung und De-Internationalisierung der Migrationsverwaltung, in: Ch. Möllers u.a. (Hrsg.), Internationales Verwaltungsrecht, 2007, S. 279, 296-299. 
zuzuweisen. ${ }^{54}$ Allerdings ist denkbar, dass funktionale Äquivalente in Gestalt von unilateralen Regelungen bestehen, die an die Staatsangehörigkeit oder den Herkunftsstaat der Migranten anknüpfen. Außerdem wirft die Gestaltung von Migration durch aufenthaltsrechtliche Privilegierungen von Angehörigen völkerrechtlicher Vertragspartner in besonderem Maße die Frage nach dem räumlichen und institutionellen Bezugsrahmen der Migrationssteuerung auf: Möglicherweise werden die abnehmenden Spielräume national definierter Privilegierungspolitiken sukzessive durch aufenthaltsrechtliche Privilegierungen als Instrument der Außenbeziehungen der EU substituiert. Der Typus des Assoziierungsabkommens könnte ein EU-spezifischer Nachfolger des Freundschafts-, Handels- und Schifffahrtsabkommens sein. Der Abschluss von „Mobilitätspartnerschaften“ mit ausgewählten Herkunftsländern im Rahmen eines auf bilaterale Kooperation ausgerichteten „Global Approach“ deutet in eine ähnliche Richtung einer Europäisierung der zwischenstaatlichen Perspektive. ${ }^{55}$

\section{Die ökonomische Perspektive}

\section{Soziale Konstruktion: Migranten als Humanressource}

Eine dritte Sichtweise betrachtet Migration als einen wirtschaftlichen Vorgang, als internationale Investition von Humanressourcen. Migranten sind nach diesem Verständnis mobile Produktionsfaktoren, die an den Prozessen des nationalen Wirtschaftssystems teilhaben, seien sie ausländische Unternehmer, zugewanderte Arbeitskräfte oder Transferleistungsempfänger. Die sozialen Rollen und ökonomischen Kategorien, die der homo oeconomicus migrans verkörpert, sind ebenso vielfältig wie die wirtschaftswissenschaftlichen und demographischen Fragestellungen, die man an Migrationsprozesse richten kann: von den Effekten der Anwerbung von Wanderarbeitnehmern unterschiedlicher Qualifikationsniveaus auf Wachstum, Beschäftigung und Einkommen über die Bedeutung von Migranten als Financiers und Klienten sozialstaatlicher Einrichtungen bis hin zu Fragen der gezielten Beeinflussung der Bevölkerungsentwicklung einer alternden und zahlenmäßig schrumpfenden Gesellschaft.

\section{Charakteristische Bauform: die Beschäftigungserlaubnis}

Wirtschaftliche Erwägungen dürften in letzter Instanz hinter einer Vielzahl von typischen Regelungen des Migrationsrechts stehen, seien es Bestimmungen des Konkurrenzschutzes für das einheimische Gewerbe, seien es Bestimmungen, die der Abwehr von Zuwanderern dienen, die der öffentlichen Fürsorge anheimfallen könnten. Die armenpolizeilichen Ausweisungs- und Abschiebemaßnahmen bilden eine der äl-

54 Siehe schon BVerwGE 36, 45, 51 ff., zum dt.-türk. Niederlassungsabkommen vom 12. Januar 1927, RGBl. II 76 und 454, BGBl. 1952 II 608.

55 Vgl. R. Parkes, EU Mobility Partnerships, EJML 11 (2009), S. 327-345. 
testen Schichten des Aufenthaltsrechts, die in zahlreichen Vorschriften des heutigen Rechts fortwirkt. Allerdings sollte man ihren Sinn und Zweck nicht auf ökonomische Motive engzuführen: Ökonomische Nützlichkeit bzw. fiskalische Interessen sind hier entstehungsgeschichtlich eng verwoben mit einer negativen Umschreibung „gefährlicher", gerade nicht nur aus wirtschaftlichen Gründen unerwünschter Migrantengruppen. ${ }^{56}$

Im historischen Archiv des Rechts findet sich ein zentrales Rechtsinstitut des Aufenthaltsrechts, das weit eindeutiger der ökonomischen Perspektive zuzuordnen ist als die Institute der Armenpolizei: es ist die Beschäftigungserlaubnis, also die staatliche Entscheidung, ob eine Arbeitsstelle mit einem ausländischen Bewerber besetzt bzw. die entsprechende Tätigkeit von ihm ausgeübt werden darf. Die auf die Erwerbstätigkeit im Inland bezogene Beschäftigungs- bzw. Arbeitserlaubnis - die rechtlichen Ausgestaltungen hinsichtlich des Genehmigungsadressaten variieren - entsteht unabhängig von der fremdenpolizeilichen Aufenthaltsgenehmigung und geht dieser als Instrument der staatlichen Migrationskontrolle in manchen Ländern voraus. ${ }^{57}$

Als Bauform der staatlichen Ordnung des Arbeitsmarkts ist auch die Beschäftigungserlaubnis ein Produkt des 20. Jahrhunderts. ${ }^{58}$ Eine Vorreiterrolle spielte das Bismarcksche Preußen, das seit den 1880er Jahren im Nordosten des Staatsgebiets ein zunächst vor allem nationalitätenpolitisch motiviertes, gegen das Anwachsen der polnischen Minderheit gerichtetes System von Registrierungs- und Genehmigungspflichten etabliert und gegen die Rekrutierungsinteressen der ostelbischen Landwirtschaft durchgesetzt hatte. ${ }^{59}$ Ein allgemeiner „Legitimationszwang“ für ausländische Arbeiter setzte sich im ersten Jahrzehnt des 20. Jahrhunderts durch, als die zur Überwachung der Saisonarbeitskräfte entwickelten Instrumente auf ganz Preußen ausgedehnt wurden. Unter der Regie der 1905 gegründeten „Deutschen Feldarbeiter-Centralstelle“ (später „Deutsche Arbeiterzentrale“) bestand seit 1909 für alle ausländischen Arbeiter die Verpflichtung, eine „Arbeiter-Legitimationskarte“ zu besitzen, die in den Grenzstellen der Arbeiterzentrale ausgefertigt wurde. Die jährlich zu erneuende Karte, in der neben dem Inhaber auch der Arbeitgeber eingetragen wurde, diente

56 Vgl. A. Fahrmeir, Paßwesen und Staatsbildung in Deutschland des 19. Jahrhunderts, HZ 271 (2000), S. 57, 67 f.; I. Reiter, Ausgewiesen, abgeschoben, 2000, S. 147-159; D. Kanstroom, Deportation Nation, 2007, S. 33-39.

57 Zur Entwicklung in Deutschland E. Weizsäcker, Vom „Inlandslegitimierungszwang“ zum „Zuwanderungsgesetz“, in: Th. Geisen (Hrsg.), Arbeitsmigration, 2005, S. 173-195.

$58 \mathrm{Zu}$ den unterschiedlichen nationalen Entwicklungswegen L. Lucassen, The Great War and the Origins of Migration Control in Western Europe and the United States, in: A. Böcker u.a. (Hrsg.), Regulation of Migration, 1998, S. 45, 55-60, F. Caestecker, The Changing Modalities of Regulation in International Migration within Continental Europe, ebd., S. 73, 79-86.

59 K. J. Bade, „Preußengänger“ und „Abwehrpolitik“, in: ders., Sozialhistorische Migrationsforschung, 2004, S. 215-302; U. Herbert, Geschichte der Ausländerpolitik in Deutschland, 2001, S. 14-37. 
sowohl zur Durchsetzung des „Rückkehrzwangs“ während der winterlichen Sperrfrist als auch zur polizeilichen Überwachung „kontraktbrüchiger“ Arbeiter. ${ }^{60}$

Systematisch ausgebaut zu einem Instrument der Arbeitsmarktpolitik wurde die Genehmigungspflichtigkeit der Ausländerbeschäftigung auch in Deutschland erst nach dem Ersten Weltkrieg. ${ }^{61}$ Sie überstand danach, bei unterschiedlichen rechtlichen Ausgestaltungen, alle politischen Systemwechsel. In der Weimarer Republik lag die Zuständigkeit für die Anwerbung, Vermittlung und Rekrutierung ausländischer Arbeitskräfte ab 1922 beim Reichsamt für Arbeitsvermittlung, das 1927 unter dem Namen Reichsanstalt für Arbeitsvermittlung und Arbeitslosenversicherung körperschaftlich verselbständigt wurde. ${ }^{62}$ Für die Ausführung waren die Landesarbeitsämter zuständig, die den antragstellenden Arbeitgebern nach Prüfung des Inländervorrangs die Erlaubnis erteilten, eine Stelle mit einem ausländischen Arbeiter zu besetzten. Über einen paritätisch besetzten Prüfungsausschuss waren auch die Vertreter der inländischen Arbeitnehmer in das Entscheidungsverfahren einbezogen. Zusätzlich mussten ausländische Arbeiter weiterhin eine Legitimationskarte bei der Deutschen Arbeiterzentrale beantragen, soweit sie nicht über eine Stichtagsregelung befreit waren. ${ }^{63}$

Das in der Bundesrepublik Deutschland errichtete System der Kontrolle der Ausländerbeschäftigung knüpfte im Wesentlichen an die Weimarer Regelungen an, allerdings ohne die formelle Einbeziehung der Gewerkschaften in das Prüfungsverfahren. ${ }^{64}$ Die doppelte Erlaubnispflicht für Arbeitgeber und Arbeitnehmer wurde 1957 durch ein generelles Verbot der Beschäftigung von Ausländern ohne Arbeitsgenehmigung ersetzt. ${ }^{65}$ Die durch die Arbeitsverwaltung erteilten Arbeitsgenehmigungen standen bis zur Reform durch das Zuwanderungsgesetz im Jahr 2005 als selbständige Verwaltungsakte neben der ordnungsbehördlichen Aufenthaltsgenehmigung. Im AufenthG hat die Beschäftigungserlaubnis die Form einer internen Zustimmung seitens der Bundesagentur für Arbeit zur Erteilung eines Aufenthaltstitels durch die Ausländerbehörde angenommen.

60 J. Oltmer, Migration und Politik in der Weimarer Republik, 2005, S. 318 f.

61 U. Herbert, Geschichte der Ausländerpolitik in Deutschland, 2001, S. 118-121; H. H. Friederichsen, Die Stellung des Fremden, S. 183-191.

62 Arbeitsnachweisgesetz vom 22. Juli 1922 - ANG (RGBl. I 657) und Gesetz über Arbeitsvermittlung und Arbeitslosenversicherung vom 16. Juli 1927 - AVAVG (RGBl. I 187).

63 J. Oltmer, Migration und Politik in der Weimarer Republik, 2005, S. 344-363; K. Dohse, Ausländische Arbeiter und bürgerlicher Staat, 1981, S. 103.

64 K. Schönwälder, Einwanderung und ethnische Pluralität, 2001, S. 217 f.; K. Dohse, Ausländische Arbeiter und bürgerlicher Staat, 1981, S. 135-144, 181; E. Weizsäcker, Vom „Inlandslegitimierungszwang“ zum „Zuwanderungsgesetz“, in: Th. Geisen (Hrsg.), Arbeitsmigration, 2005, S. 173, $180 \mathrm{f}$.

65 \55 AVAG i.d. Neufassung vom 23.12.1956, BGBl. I 1018. 


\section{Anliegen der Migrationssteuerung: Wohlstandsmehrung und Schutz des inländischen Arbeitsmarktregimes}

Die sozialen Konstruktionen von Migration, die hier zusammenfassend als ökonomische Perspektive bezeichnet werden, zeichnet erfahrungsgemäß eine Affinität zum Utilitarismus als normativer Grundhaltung aus. Aus diesem Blickwinkel werden Migrationsvorgänge vor allem hinsichtlich ihres wirtschaftlichen Nutzens für die Aufnahmegesellschaft bewertet. Nicht anders als bei den anderen Perspektiven ist diese Verschwisterung ein Produkt diskursiver Praxis und nicht schon mit einer ökonomischen Sicht auf das Migrationsgeschehen notwendig verbunden. ${ }^{66}$ Die Offenheit für liberale wie für restriktive Politiken ist besonders markant, wenn man nach den wirtschaftlichen Interessenlagen unterschiedlicher Gruppen in den Zielländern differenziert und die aus ihnen resultierenden heterogenen Forderungen in den Blick nimmt. ${ }^{67}$

Gibt es ein übergreifendes Anliegen, das die Steuerung der Migration aus einer ökonomischen Perspektive auszeichnet? Man mag dies bezweifeln, ist diese Perspektive doch besonders anfällig dafür, dass sich in den einschlägigen migrationspolitischen Forderungen je partikuläre und konjunkturell schwankende Interessen artikulieren. Immerhin ist das Erreichen von Steuerungszielen für eine Migrationspolitik, die Migranten in erster Linie als mobilen Produktionsfaktor ansieht, regelmäßig der erklärte Zweck ihrer Interventionen: Ihr gemeinsamer Nenner ist, allgemein formuliert, die Wohlstandsmehrung.

Die Migrationspolitik ist aber nicht einfach eine Unterabteilung der allgemeinen Wirtschaftspolitik, häufig weist sie eine besondere Beziehung zur Arbeitsmarktpolitik und den sie prägenden Interessenlagen und institutionellen Arrangements auf. Die Mehrzahl der erwerbstätigen Migranten gehört nicht zum Kreis der transnationalen Wirtschaftseliten: Arbeitswanderer sind typischerweise Wanderarbeiter, also abhängig Beschäftigte, sei es auf dem regulären Arbeitsmarkt, sei es im informellen Sektor. Ein übergreifendes Anliegen der Migrationssteuerung aus einer ökonomischen Perspektive bildet vor diesem Hintergrund die Funktionsfähigkeit des etablierten Systems der Arbeitsbeziehungen, also der Schutz der inländischen Arbeitsverfassung vor einer möglichen Desintegration durch internationale Migrationsprozesse.

Um diesen Zusammenhang zu erläutern, ist erneut ein Blick auf die Genese des Beschäftigungserlaubnisrechts hilfreich. Die Etablierung einer allgemeinen Arbeitsgenehmigungspflicht in der Weimarer Republik fällt nicht zufällig zusammen mit der Konstituierung eines nationalen Regimes der industriellen Arbeitsbeziehungen, das auf einem Kompromiss zwischen Kapital und Arbeit beruht. ${ }^{68}$ Das Migrationsregime

66 Vgl. z.B. J. W. Moses, International Migration, 2006, S. 18-24, 123-131.

67 Instruktiv T. Jacoby, Immigration Nation, Foreign Affairs 85 (2006), S. 50-65. 
bildet einen wesentlichen Bestandteil der nationalen Arbeitsverfassung, die diesen Kompromiss verrechtlicht und zugleich den ordnenden Zugriff auf den Arbeitsmarkt verstärkt. ${ }^{69}$ Aus Sicht der Unternehmer besteht grundsätzlich das Interesse an einer möglichst freien Rekrutierung im Ausland zwecks Erschließung eines erweiterten, mobilen Arbeitskräftereservoirs. ${ }^{70}$ Dies gilt verstärkt in Zeiten der Vollbeschäftigung bzw. bei expandierender Arbeitskräftenachfrage, um den Anstieg der Löhne zu dämpfen, sodass die Ausländerbeschäftigung als konjunkturelles Ausgleichsinstrument fungiert. ${ }^{71}$ Aus Sicht der Arbeitnehmer dagegen verbindet sich mit der unreglementierten Rekrutierung ausländischer Arbeitskräfte die Befürchtung, dass örtliche Tarife und Beschäftigungsbedingungen unterlaufen und unorganisierte Wanderarbeiter als Streikbrecher eingesetzt werden. Vor diesem Hintergrund hat die Durchsetzung eines Erlaubnisvorbehalts für die Ausländerbeschäftigung einen disziplinierenden Effekt für die Arbeitgeber und begünstigt die Herausbildung eines stabilen Systems industrieller Arbeitsbeziehungen. Die Erlaubnispflicht ermöglicht es nicht nur, volkswirtschaftliche Gesichtspunkte in die Rekrutierungspraxis einzustellen, sondern auch die Interessen der inländischen Arbeitnehmer.

Entsprechend beruhte der historische Kompromiss mit den Gewerkschaften, der in der Weimarer Republik entwickelt und in der Bundesrepublik mit Beginn der Anwerbepolitik erneuert wurde, im Wesentlichen auf zwei Säulen: erstens die Geltung gleicher Tarife und betrieblicher Beschäftigungsbedingungen für inländische und ausländische Arbeitnehmer, zweitens die Durchführung einer arbeitsplatzbezogenen Vorrangprüfung, die der Durchsetzung des Inländerprimats bei der Besetzung freier Stellen dient. Die ordnungsrechtliche Kontrolle der Erwerbsmigration gehört so zu den Kernbestandteilen eines nationalen Arbeitsmarktregimes, das Erwerbsmigration in Abhängigkeit von der Bedarfslage einzelner Sektoren ermöglichen will, ohne den Kompromiss zwischen Kapital und Arbeit aufzukündigen. ${ }^{72}$

Eine gewisse Widersprüchlichkeit ist diesem Regime insofern inhärent, als die Geltung gleicher Beschäftigungsbedingungen auch die Einbeziehung in die sozialen Sicherungssysteme beinhaltet. Die hieraus erwachsenen Ansprüche, namentlich auf Transfer- und Vermittlungsleistungen im Fall der Arbeitslosigkeit, können forcierte Rückführungspolitiken in Zeiten des konjunkturellen Abschwungs erschweren und so die Funktion der Ausländerbeschäftigung als Konjunkturpuffer schwächen. ${ }^{73}$ Eine

68 Zur zeitgenössischen Sicht siehe J. Bast, Totalitärer Pluralismus, 1999, S. 52-59.

69 U. Herbert, Geschichte der Ausländerpolitik in Deutschland, 2001, S. 121-123; K. J. Bade, Europa in Bewegung, 2000, S. $257 \mathrm{f}$.

70 K. Dohse, Ausländische Arbeiter und bürgerlicher Staat, 1981, S. 178.

71 U. Herbert, Geschichte der Ausländerpolitik in Deutschland, 2001, S. 211.

72 Zum sog. immigration/welfare paradox siehe G. Brochmann, The Mechanism of Control, in: dies./ T. Hammar (Hrsg.), Mechanisms of Immigration Control, 1999, S. 1, 15.

73 B. Sonnenberger, Verwaltete Arbeitskraft, in: J. Oltmer (Hrsg.), Migration steuern und verwalten, 2003, S. 145, 169-172. 
offene Frage schließlich ist es, wie die weitere Europäisierung des Migrationsrechts auf die in vieler Hinsicht national verfassten Regimes der Arbeitsbeziehungen zurückwirkt und wie sich die Anliegen der ökonomischen Perspektive in dem neuen Mehrebenen-Bezugsrahmen artikulieren. ${ }^{74}$

\section{E. Die kulturelle Perspektive}

\section{Soziale Konstruktion: Migranten als die Anderen}

Der Anknüpfungspunkt für eine weitere Perspektive ist die alltagskulturelle Praxis von Migranten bzw. der jeweiligen ethnisch-sozialen Gemeinschaft („migrant community “): ihre Sprache, ihre Religion, ihre Sitten und Gebräuche, oder für die hiesigen Zwecke kurz: ihre Kultur. Internationale Migrationsprozesse erscheinen hier als Kulturtransfers und die Migranten als Träger einer Kultur, die sich von der der Einheimischen unterscheidet. ${ }^{75}$ Bei der kulturellen Perspektive geht es also um - zugeschriebene oder tatsächliche - Nähe bzw. Distanz. Diese bemisst sich nicht allein nach objektiven Kriterien, denn bei der Markierung von kulturellen Differenzen spielen kontingente Muster der Selbst- und Fremdwahrnehmung eine Rolle, die sich im Zeitablauf ändern. ${ }^{76}$ Die Fluidität von kulturellen Zuschreibungen belegt exemplarisch die Darstellung türkischer Gastarbeiter in den deutschen Medien, die in den 1960er und 1970er kaum anders als ihre italienischen oder spanischen Kollegen als „südländisch“ wahrgenommen wurden, und erst seit den 1980er Jahren verstärkt als „islamisch“. 77

Die Schärfe von Konflikten über migrationspolitische Fragen rührt oftmals von einer Option der Konfliktparteien für diese Perspektive. Hier werden sensible Identitätsfragen verhandelt: Migranten personifizieren das Fremde, in Abgrenzung zu dem sich das Eigene, der normative Entwurf davon, wer „wir“ sind, konstituiert. ${ }^{78}$ Die Migranten sind „die Anderen“, die der Aufnahmegesellschaft den Spiegel vorhalten und fragen: Wer seid ihr, wie wollt ihr leben, was ist euch heilig? Die Antworten können je nach der kulturellen Identität des Angesprochenen höchst unterschiedlich ausfallen, und so ist auch diese soziale Konstruktion von Migration nicht auf eine bestimmte Migrationspolitik festgelegt: Ein migrationsbedingter Kulturtransfer kann gleichermaßen als bedrohliche „Überfremdung“ oder als bereichernder Beitrag zu „kultureller Vielfalt“ empfunden werden. Jedoch zeigt die Erfahrung, dass die kulturelle Perspektive eine hohe Affinität zu einer restriktiven Migrationspolitik auf-

74 Vgl. F. Rödl, Arbeitsverfassung, in: A. von Bogdandy/J. Bast (Hrsg.), Europäisches Verfassungsrecht, 2009, S. 855, 860, $903 \mathrm{f}$.

75 L. Orgad, ,Cultural Defence‘ of Nationals, ELJ 15 (2009), S. 719, 737.

76 G. Britz, Kulturelle Rechte und Verfassung, 2000, S. 90 f., 108.

77 K. Hunn, „Nächstes Jahr kehren wir zurück ...“, 2005, S. 492-506.

78 B. Waldenfels, Topographie des Fremden, 1997, S. 145-165; zur Wiedererkennung verdrängter Anteile der eigenen Identität im Fremden siehe J. Kristeva, Fremde sind wir uns selbst, 1990, S. 184-210. 
weist. ${ }^{79}$ Für einwanderungsbezogenen Restriktionismus kann es viele Motive und Gründe geben: wohlstandschauvinistische ebenso wie sozialpolitische, benevolente wie egoistische, rationale wie irrationale. Seine politisch-soziale Triebfeder aber ist oftmals ein kulturell aufgeladener Nationalismus: Einwanderer werden als nicht integrierbar und nicht integrationsbereit, als Bedrohung der eigenen Lebensweise und nationalen Eigenarten angesehen. ${ }^{80}$ Dabei dient die Rede von „kultureller Differenz“ nicht selten als ein verschleierndes Kürzel für Imaginationen kultureller Überlegenheit und für rassistische Stereotypisierungen. ${ }^{81}$

\section{Charakteristische Bauform: der Sprachtest}

Welches sind die typischen Bauformen, die dieses Verständnis von Migration verkörpern? Obwohl Gesichtspunkte kultureller Differenz im politischen Diskurs über Migrationsfragen stark präsent und politisch wirkungsmächtig sind, ${ }^{82}$ tritt die kulturelle Perspektive im Recht oftmals nicht offen zu tage. Es erfordert dann Einsichten in politische Kontexte und administrative Anwendungspraktiken, um aufzuhellen, ob sich hinter einer neutral formulierten Regelung ein kulturell aufgeladener Blick auf Migranten verbirgt.

Als exemplarische Bauform kommen Sprachtests in Betracht. Bei diesen handelt es sich um rechtlich fixierte Integrationsanforderungen, die die Erteilung eines (günstigeren) Aufenthaltstitels davon abhängig machen, dass der Antragsteller ein bestimmtes Sprachniveaus in einer vorgegebenen Sprache beherrscht. Die Sprache der Einheimischen dient als Schibboleth, als linguistisches Erkennungszeichen einer sozialen Gruppe. ${ }^{83}$ Der individuelle Erwerb von Sprachkompetenzen in Wort und Schrift wird zum Gradmesser der Bereitschaft zur sozio-kulturellen Integration, denn wer sich nicht bemüht, die Verkehrssprache zu erlernen, nimmt nicht am interkulturellen Dialog und (idealiter) am gemeinsamen Diskurs über die öffentlichen Angelegenheiten teil, will also auf Dauer nicht zu „uns“ gehören.

Das Testen von Sprachkompetenzen für aufenthaltsrechtliche Zwecke ist eine noch recht junge Bauform, jedenfalls im europäischen Rechtsvergleich. Sie wurde zuerst

79 Eingehend zur „cultural dimension“ in der Einwanderungspolitik A. R. Zolberg/Long L. W., Why Islam Is Like Spanish: Cultural Incorporation in Europe and the United States, Politics \& Society 27 (1999), S. 5-38.

80 A. R. Zolberg, Matters of State, in: Ch. Hirschman u.a. (Hrsg.), The Handbook of International Migration, 1999, S. 71, 84 f.; A. Somek, Wie erklären wir potentiellen Einwanderern, was wir unseren Landsleuten schulden?, in: A. Märker/St. Schlohfeldt (Hrsg.), Was schulden wir Flüchtlingen und Migranten?, 2002, S. 171, $174 \mathrm{f}$.

81 Zum Konzept des kulturellen Rassismus siehe St. Hall, Die Frage der kulturellen Identität, in: ders., Rassismus und kulturelle Identität, 1994, S. 180, 207 f., 213-222.

82 Exempl. H. Mahnig, Between Economic Demand and Popular Xenophobia, in: A. Böcker u.a. (Hrsg.), Regulation of Migration, 1998, S. 174, 175-183.

83 T. McNamara, 21st Century Shibboleth: Language Tests, Identity and Intergroup Conflict, Language Policy 4 (2005), S. 351, 352-356, vgl. Buch der Richter 12,5-6. 
in Deutschland entwickelt, und zwar in der sog. Verfestigungsregelung, die seit dem 7. Juli 1978 in der Allgemeinen Verwaltungsvorschrift zum AuslG 1965 niedergelegt war. ${ }^{84}$ Darin eröffnete die Bundesregierung der ersten Generation der Arbeitsmigranten nach Verkündung des Anwerbestopps die Aussicht auf ein Daueraufenthaltsrecht, knüpfte die Erteilung des entsprechenden Titels (einer „Aufenthaltsberechtigung “, nach damaliger Terminologie) aber unter anderem an „ausreichende Kenntnisse der deutschen Sprache“. Nach der Gesetzesauslegung des Bundesverwaltungsgerichts aus dem Jahr 1987 erforderte dies, sich in einer deutschen Umgebung sprachlich ohne nennenswerte Probleme zurechtfinden zu können. ${ }^{85}$ Für die etwas schwächere „unbefristete Aufenthaltserlaubnis“ genügte es, sich „auf einfache Art in deutscher Sprache mündlich verständlich machen [zu] können". Seitdem sind Sprachtests bei der Verfestigung des Aufenthaltsstatus ein fester Bestandteil des deutschen Rechts. Das AufenthG heute verlangt durchgängig ,ausreichende Kenntnisse“ der deutschen Sprache, bevor ein unbefristeter Aufenthaltstitel erworben werden kann. Nach der gesetzlichen Festlegung entspricht dies dem Sprachniveau B1 des Gemeinsamen Europäischen Referenzrahmens für Sprachen, verlangt also selbständige Sprachverwendung in Wort und Schrift.

Es mag überraschen, dass dieser deutsche Ansatz erst in den letzten Jahren in anderen europäischen Ländern Nachahmer gefunden hat, etwa in Frankreich. ${ }^{86}$ Nach rechtsvergleichenden Untersuchungen Ende der 1990er Jahre war sonst lediglich in Ungarn für den Erwerb eines Daueraufenthaltsstatus ein Sprachtest vorgesehen. ${ }^{87}$ Die Trendwende setzte zwischen 2000 und 2004 ein. ${ }^{88}$ Die EU-Daueraufenthaltsrichtlinie von 2003 erlaubt den Mitgliedstaaten, besondere „Integrationsanforderungen“ für den Statuserwerb aufzustellen, was mittlerweile von der Mehrzahl der teilnehmenden 24 Mitgliedstaaten in der einen oder anderen Weise für verpflichtende Integrationskurse sowie Sprach-, Wissens- und Sozialkompetenztests genutzt wird. ${ }^{89}$ Auch jenseits der Aufenthaltsverfestigung haben Sprachtests vermehrt aufenthaltsrechtliche Bedeutung erlangt. Sie werden teilweise schon auf neuankommende Migranten angewendet, insbesondere verlangen einige Staaten, darunter die Bundesrepublik, beim Ehegattennachzug den Nachweis von Kenntnissen der inländischen Amtssprache. ${ }^{90} \mathrm{Im}$ internationalen Vergleich finden sich solche Voraussetzungen traditionell eher im

84 GMBl. 1978, 368.

85 BVerwGE 77, 188, $198 \mathrm{f}$.

86 Ausf. S. Carrera, In Search of the Perfect Citizen?, 2009, S. 291-349.

87 K. Groenendijk, Legal Concepts of Integration in EU Migration Law, EJML 6 (2004), S. 111, 116.

88 K. Groenendijk, The Legal Integration of Potential Citizens, in: R. Bauböck u.a. (Hrsg.), Aquisition and Loss of Nationality, Bd. 1, 2006, S. 385, 397-400.

89 Ausf. D. Acosta, The Long-Term Residence Status as a Subsidiary Form of EU Citizenship, 2011.

90 Im Detail R. van Oers/E. Ersbøll/D. Kostakopoulou (Hrsg.), A Re-definition of Belonging?, 2010; zur Vorreiterrolle der Niederlande siehe L. F. M. Besselink, Integration and Immigration, in: E. Guild u.a. (Hrsg.), Illiberal Liberal States, 2009, S. 241-257. 
Staatsangehörigkeitsrecht, und zwar als Anforderung an die Einbürgerung. ${ }^{91}$ In Europa hat also sukzessive eine Anreicherung des Aufenthaltsrechts mit einem traditionellen Institut des Staatsangehörigkeitsrechts stattgefunden. Nur in Deutschland war der Rezeptionsweg umgekehrt, wie sich in der Aufnahme des Erfordernisses der „ausreichenden Kenntnisse der deutschen Sprache“ in die gesetzlichen Einbürgerungsvoraussetzungen nach der Reform des Staatsangehörigkeitsrechts von 1999 zeigt. $^{92}$

Die Archäologie des Rechts fördert aber noch eine ältere, verschüttete Schicht von Sprachtests zutage - wenn die modernen Sprachtests dieses problematische Erbe denn antreten wollen. Bei der historischen Spurensuche nach der kulturellen Perspektive im Aufenthaltsrecht stößt man auf das rassistische Erbe der klassischen Einwanderungsländer USA, Kanada, Australien und Neuseeland, die bis in die 1960er Jahre (Australien bis 1973) diskriminierende Ausschlusskriterien gegenüber nicht-europäischen Einwanderern formulierten. Aristide R. Zolberg hat diese Regelungen metaphorisch als Antichinesische Mauer („The Great Wall against China“) bezeichnet. Die Bezeichnung nimmt Bezug auf die unverhüllt rassistische Einwanderungsgesetzgebung der USA seit den 1880er Jahren, die sich zunächst gegen chinesische Arbeiter richtete. ${ }^{93}$ Zolberg und andere unterstreichen den epochalen Charakter des identitätsbezogenen Restriktionismus, der sich um die Wende zum 20. Jahrhundert formte und der für Jahrzehnte in zahlreichen Ländern zur gestaltungsmächtigen politischen Kraft aufstieg. ${ }^{94}$ Die rechtlichen Instrumente zur Implementierung dieser Politik waren vielgestaltig und innovativ. So führten die USA 1921/24 ein Quotensystem ein, das „,asiatische“ Einwanderer ganz ausschloss und die übrigen je nach der „nationalen Herkunft“ zahlenmäßig begrenzte. Der kanadische Immigration Act von 1910, der mit sprachlichen Modifikationen bis 1952 in Kraft war, untersagte in direkter Anknüpfung an das Merkmal der „Rasse“ den Zugang von „immigrants belonging to any race deemed unsuited to the climate or requirements of Canada“.

91 Vgl. H. F. Schiffman, Language, Language Policy, and Citizenship, in: J. V. Ciprut (Hrsg.), The Future of Citizenship, 2008, S. 75, 85-93.

92 U. Davy, Einbürgerung in Deutschland, DV 41 (2008), S. 31, 35-39.

93 A. R. Zolberg, The Great Wall against China, in: J. Lucassen/L. Lucassen (Hrsg.), Migration, Migration History, History, 2005, S. 291, 304-310; zur Entstehung der sog. Chinese Exclusion Acts und deren Bestätigung durch den US Supreme Court siehe L. E. Salyer, Laws Harsh as Tigers, 1995, S. 132, und H. Motomura, Americans in Waiting, 2006, S. 15-31; zum schwierigen Prozess der Überwindung dieser Tradition nach 1965 siehe ebd., S. 123-135, 183-188.

94 A. R. Zolberg, Global Movements, Global Walls, in: Wang Gungwu (Hrsg.), Global History and Migrations, 1997, S. 279, 293-303; D. Gabaccia, The, Yellow Peril' and the ,Chinese of Europe', ebd., S. 177, 189-196; P. Weil, Races at the Gates, in: A. Fahrmeir u.a. (Hrsg.), Migration Control in the North Atlantic World, 2003, S. 271, 273-280; zu Entstehung und Hintergründen der Politik eines „White Canada“ bzw. „White Australia“ F. Hawkins, Critical Years in Immigration, 1989, S. 8-30. 
Als ein Instrument zur Errichtung „Antichinesischer Mauern“ im Einwanderungsrecht wurden schließlich auch Sprach- bzw. Alphabetisierungstests eingesetzt, in denen rassistische Motive und die traditionelle Abwehr ungebildeter, verarmter Einwanderer eine enge Verbindung eingingen. ${ }^{95}$ Entwickelt wurden solche Sprachtests um die Jahrhundertwende an der Peripherie des Britischen Empire, wo der Wille zur Abwehr „asiatischer“ (= nicht-europäischer) Einwanderer auf Seiten der selbstverwalteten „weißen“ Siedlerkolonien mit den außenpolitischen Rücksichtnahmen der Krone, vor allem gegenüber Japan, abgeglichen werden musste. Erstmals erprobt wurde ein solcher Test in der südafrikanischen Kolonie Natal im Jahr 1897, um unerwünschte Einwanderer vom indischen Subkontinent, die von imperialen Freizügigkeitsrechten profitierten, auszuschließen. ${ }^{96}$ Der sog. Natal Dictation Test ging dann, leicht modifiziert, in den Immigration Restriction Act von 1901 des jungen Commonwealth of Australia ein und blieb bis 1957 als Schlüsselinstrument einer Politik des „White Australia“ in Kraft. ${ }^{97}$ Verlangt wurde ein 50 Worte umfassendes Diktat in einer „europäischen “, später scheinbar neutral in einer „vorgeschriebenen“ Sprache. Durch die flexible Stellgröße einer im Zweifel durch den Einwanderungsbeamten ausgewählten Sprache und mangels Kontrolle durch eine neutrale Instanz ermöglichte er eine außenpolitisch diskrete und zugleich unnachgiebige Verfolgung der rassistischen Ausschlusspolitik. ${ }^{98}$

\section{Anliegen der Migrationssteuerung: Achtung kultureller Identitäten und Wahrung der „kulturellen Kompromisse“}

Ungeachtet dieses dunklen Erbes gilt es festzuhalten, dass Sprachtests für Zuwanderer keine per se illegitimen Mittel der Migrationssteuerung sind, so wie insgesamt die kulturelle Perspektive im Aufenthaltsrecht nicht mit krypto-rassistischer Ausgrenzung gleichgesetzt werden darf. ${ }^{99}$ Auf einen Nenner gebracht dient die Sensibilität des Rechts für die kulturelle Dimension der Migration dazu, die Identitäten der Beteiligten, sowohl auf Seiten der Migranten als auch der Alteingesessenen, in die rechtliche Gestaltung einzustellen und nicht ohne gute Gründe zu missachten. Man

95 Rechtsvergl. B. Voigt, Gesetzliche Schranken für Ausländer bei der Einwanderung in Deutschland, 1932, S. 21-23.

96 M. Lake, From Mississippi to Melbourne via Natal, in: A. Curthoys/M. Lake (Hrsg.), Connected Worlds, 2005, S. 209, 219-221.

97 Ebd., S. 222-229; F. Hawkins, Critical Years in Immigration, 1989, S. 14-16, 33.

98 Zur Anwendungspraxis, die die Regelung zunächst als Bildungsklausel missverstand, siehe illustrativ T. McNamara, 21st Century Shibboleth, Language Policy 4 (2005), S. 351, 356-360. Vgl. die wohlwollende Darstellung der fremdenrechtlichen „Rassengesetze“ in der staatsrechtlichen Monographie Hans von Frischs (Das Fremdenrecht, 1910, S. 102-114), der den Bogen von den „Antichinesengesetzen“ der USA und der britischen Kolonien zu den europäischen Maßnahmen gegen „, herumziehende Zigeuner" schlägt.

99 Für eine dezidierte Verteidigung siehe M. Weiner, The Global Migration Crisis, 1995, S. 83-92, 140142; abwägend A. Märker, Europäische Zuwanderungspolitik und globale Gerechtigkeit, 2005, S. $118-122$. 
könnte also von der Aufgabe der kulturell sensiblen Institute des Aufenthaltsrechts sprechen, die etablierten „kulturellen Kompromisse“ einer Gesellschaft zu wahren. ${ }^{100}$ Migrationssteuerung aus kultureller Perspektive blickt auf die Folgen der Einwanderung für den gesellschaftlichen und den staatlichen Umgang mit religiöser und sprachlicher Pluralität und stellt auf die - befürchtete oder erhoffte - Neuverhandlung der entsprechenden Arrangements ab. ${ }^{101}$ Sie vorverlagert in das Aufenthaltsrecht, was in anderen Kontexten als Probleme der internen Verfassung moderner, kulturell heterogener Gesellschaften thematisiert wird. ${ }^{102}$

Allerdings steht die Sichtbarkeit der kulturellen Dimension im Aufenthaltsrecht vor einem Dilemma. Einerseits entsteht das Bedürfnis nach rechtlicher Institutionalisierung kulturell sensibler Bauformen wie den Sprachtests erst, sobald kulturelle Selektivität in der Migrationspolitik rechtfertigungsbedürftig wird, also wenn der religiöse Dissenter oder eine fremdartige Lebensweise nicht mehr ohne Weiteres als Ordnungsproblem definiert werden können. Ihre Präsenz im Recht ist also zum Teil ein Produkt der rechtsstaatlichen Einhegung der Ausländerpolizei.

Andererseits ist Migrationssteuerung aus der Perspektive kultureller Differenz unter den Bedingungen liberaler Rechtsstaatlichkeit tendenziell problematisch. Erstens gerät sie leicht in den Verdacht, entlang verbotener Merkmale zu diskriminieren und ist dann nicht nur politisch, sondern auch verfassungs- und völkerrechtlich delegitimiert. Kritische Rückfragen ergeben sich selbst bei aufenthaltsrechtlichen Privilegierungen kultureller Minderheiten, etwa bei der Auswahl von Kontingentflüchtlingen aufgrund der Zugehörigkeit zu einer bestimmten Religionsgemeinschaft. ${ }^{103}$ Zweitens hält der liberale Verfassungsstaat mit den Grundrechten einen Abwehranspruch gegen aufgedrängte Identitäten und überzogene Integrationserwartungen bereit ${ }^{104}$ und setzt damit einer auf Wahrung bzw. Herstellung kultureller Homogenität gerichteten

100 Zum Begriff siehe A. R. Zolberg, Matters of State, in: Ch. Hirschman u.a. (Hrsg.), The Handbook of International Migration, 1999, S. 71, 84; skeptisch gegenüber dem rechtlichen Schutz der Anliegen der Mehrheitskultur G. Britz, Kulturelle Rechte und Verfassung, 2000, S. 281-282, 300-301; für die Gegenansicht A. Somek, Wie erklären wir potentiellen Einwanderern, was wir unseren Landsleuten schulden?, in: A. Märker/St. Schlohfeldt (Hrsg.), Was schulden wir Flüchtlingen und Migranten?, 2002, S. 171, 185-189.

101 A. R. Zolberg/Long L. W., Why Islam Is Like Spanish: Cultural Incorporation in Europe and the United States, Politics \& Society 27 (1999), S. 5, 13-28.

102 Siehe einerseits B.-O. Bryde, Wandlungen des Rechtssystems in der Einwanderungsgesellschaft, in: ders. (Hrsg.), Das Recht und die Fremden, 1994, S. 7-13; G. Frankenberg, Die Verfassung der Republik, 1997, S. 94-97; R. Nickel, Gleichheit und Differenz in der vielfältigen Republik, 1999, S. $56-$ 66; H. Bielefeldt, Menschenrechte in der Einwanderungsgesellschaft, 2007, S. 57-73, 183-193, andererseits A. Uhle, Innere Integration, in: J. Isensee/P. Kirchhof (Hrsg.), HStR IV, 3. Aufl. 2006, $\$ 82$ Rn. 38; St. Luft, Staat und Migration, 2009, S. 259 ff. et passim; J. Isensee, Integration mit Migrationshintergrund, JZ 2010, S. 317-327.

103 Zum Resettlement von Flüchtlingen, die religiösen Minderheiten angehören, siehe N. Allenberg, Die Aufnahme irakischer Flüchtlinge in Deutschland, Asyl 4/2009, S. 10-13.

104 A. von Bogdandy, Europäische und nationale Identität, VVDStRL 62 (2003), S. 156, 178-181; vgl. in diesem Sinne BVerfGE 76, 1, 53 - Familiennachzug I. 
Migrationssteuerung enge Grenzen. Dies hat jedoch zur Folge, dass an die Stelle explizit kultursensibler Regelungen andere, scheinbar neutrale Kriterien treten, was die rationale Thematisierung der aufgeworfenen Identitätsfragen und der möglichen Rückwirkungen von Einwanderung auf die „kulturellen Kompromisse“ einer Gesellschaft erschwert. Drittens birgt die Europäisierung der Migrationssteuerung das Potenzial zu massiven Erschütterungen für die kulturelle Perspektive auf das Migrationsgeschehen. Kritisch wird sein, welche Spielräume für nationale Identitätspolitiken und die Stabilisierung der lokalen kulturellen Kompromisse verbleiben - in einer EU, die sich selbst und ihren Umgang mit Drittstaatsangehörigen kaum plausibel auf die Prämisse kultureller Homogenität der Unionsbürger gründen kann. ${ }^{105}$

\section{F. Die individualrechtliche Perspektive}

\section{Soziale Konstruktion: Migranten als Rechtssubjekte}

Als letztes ist hier ein individualrechtliches Verständnis von Migration anzuführen. Der analytische Bezugspunkt dieser Perspektive sind die Rechtsgüter und Interessen von Menschen, die sich in einer Migrationssituation befinden; sie werden als Personen, als Inhaber von subjektiven Rechten wahrgenommen. Im englischsprachigen Diskurs ist die Bezeichnung als „rights-based approach“ anzutreffen, ${ }^{106}$ zuweilen auch - mit gewissen Unschärfen, dazu sogleich - als „human rights approach“ bzw. „menschenrechtliche Ausrichtung“. 107

\section{Charakteristische Bauform: das Refoulementverbot}

Ein identifizierendes Merkmal der individualrechtlichen Perspektive bildet die Ablösung der Rechtsstellung der Migranten von der Staatsangehörigkeit, dem Schlüsselinstitut der zwischenstaatlichen Perspektive. Die Suche nach typischen Bauformen setzt deshalb bei den völkerrechtlichen Entwicklungen nach dem Zweiten Weltkrieg an, die zur Anerkennung universeller Menschenrechte für Migranten führten, und setzt sich im Bereich des Flüchtlingsrechts fort.

Die älteren Gebote fremdenrechtlicher Mindeststandards sind eine der völkerrechtlichen Quellen, aus denen die universellen Menschenrechte, wie sie die Allgemeine Erklärung von 1948 formuliert, hervorgegangen sind. ${ }^{108}$ Das revolutionär Neue an der menschenrechtlichen Sichtweise auf Migranten bestand darin, sie unabhängig

105 F. Hanschmann, Der Begriff der Homogenität in der Verfassungslehre und Europarechtswissenschaft, 2008, S. 287-295; anders J. Isensee, Integration mit Migrationshintergrund, JZ 2010, S. 317, 319.

106 Etwa A. Wiesbrock, Legal Migration to the European Union, 2010, S. 5 f., m.w.N.

107 Zu den Prämissen des Ansatzes siehe D. Thym, Schutz des Aufenthalts zwischen polizeilicher Herkunft und menschenrechtlicher Neuausrichtung, in: F. Arndt u.a. (Hrsg.), Freiheit, Sicherheit, Öffentlichkeit, 2009, S. 221, 225-228; R. Keil, Freizügigkeit, Gerechtigkeit, demokratische Autonomie, 2009, S. 27-58.

108 R. B. Lillich, The Human Rights of Aliens in Contemporary International Law, 1984, S. 5-37. 
von der Zugehörigkeit zu einer politischen Gemeinschaft als Rechtssubjekte anzusprechen. ${ }^{109}$ Die weiteren Bemühungen um menschenrechtliche Garantien für Migranten haben sich vor allem auf Rechte in der Migrationssituation konzentriert und aufenthaltsrechtliche Fragen zumeist ausgeklammert. ${ }^{110}$ Besondere Aufmerksamkeit erfuhren die migrationspezifischen Gefährdungslagen von Wanderarbeitnehmern. ${ }^{111}$ Dass reguläre Migranten darüber hinaus menschenrechtliche Garantien im Hinblick auf ihren Aufenthaltsstatus genießen, ist eine jüngere Entwicklung, die sich vor allem auf den europäischen Rechtsraum bezieht, mit Einschränkungen wohl auch auf Lateinamerika. ${ }^{112}$ Dieser zweite Schub der menschenrechtlichen Aufwertung privater Migrationsinteressen, dessen Ansätze auf die 1970er Jahre zurückgehen, lässt sich als eine Reaktion auf die gesellschaftspolitischen Folgen der postkolonialen und erwerbsbezogenen Einwanderungen in die Industriestaaten West- und Nordeuropas in den 1950er und 1960er Jahren verstehen. ${ }^{113}$ Ausgehend von der sozialen Situation der ehemaligen Wanderarbeitnehmer entwickelte sich der Gedanke des rechtlichen Schutzes von Inklusionserwartungen, der sich in der Judikatur der Europäischen Menschenrechtskommission und des EGMR zu bi-nationalen Ehen, zum Familiennachzug und zum Ausweisungsschutz für Einwanderer der zweiten Generation niedergeschlagen hat. Seit den 1990er Jahren schließlich sind in der Rechtsprechung des EGMR sowohl das Zusammenleben mit Familienangehörigen als auch die sozialen Bindungen am Aufenthaltsort als menschenrechtlich geschützte Interessen anerkannt, mit starken aufenthaltsrechtlichen Implikationen. ${ }^{114}$

Der rechtshistorische Geburtsort der individualrechtlichen Perspektive im Aufenthaltsrecht - und bis heute ihr ideologisches Kraftzentrum - liegt jedoch auf dem Gebiet des internationalen Flüchtlingsschutzes: In den völkerrechtlichen Regelungen zugunsten von Flüchtlingen und Staatenlosen wurden die Einzelnen erstmals losge-

109 Dazu hier nur N. Bobbio, Das Zeitalter der Menschenrechte, 1998, S. 12 f.; J. von Bernstorff, The Changing Fortunes of the Universal Declaration of Human Rights, EJIL 19 (2008), S. 903-924.

110 Im Überblick J. Fitzpatrick, The Human Rights of Migrants, in: T. A. Aleinikoff/V. Chetail (Hrsg.), Migration and International Legal Norms, 2003, S. 169-184; W. Kälin/J. Künzli, Universeller Menschenrechtsschutz, 2. Aufl. 2008, S. 544-570; D. Weissbrodt, The Protection of Non-Citizens in International Human Rights Law, in: R. Cholewinski u.a. (Hrsg.), International Migration Law, 2007, S. 221-235.

111 Exempl. R. Cholewinski, Migrant Workers in International Human Rights Law: Their Protection in Countries of Employment, 1997; ders., The Rights of Migrant Workers, in: R. Cholewinski u.a. (Hrsg.), International Migration Law, 2007, S. 255-274.

112 Vgl. J. M. Casal Hernández, Migration und internationaler Menschenrechtsschutz, ZaöRV 68 (2008), S. 491-508.

113 A. Farahat, Progressive Inklusion und statische Zuordnung, Ms. (2010), Kap. 2. C. III.; U. Davy, Überregionales und regionales Völkerrecht, in: dies. (Hrsg.), Die Integration von Einwanderern, 2001, S. 37, 74-76.

114 C. Murphy, The Concept of Integration in the Jurisprudence of the European Court of Human Rights, EJML 12 (2010), S. 23-43. 
löst vom Band der Staatsangehörigkeit als Personen und Inhaber von Rechten anerkannt. ${ }^{115}$

Als Kandidat für eine typische Bauform kommt zunächst der Flüchtlingspass in Frage. Das Konzept entstand in der Zwischenkriegszeit mit dem sog. Nansen-Pass. Das nach dem Hohen Flüchtlingskommissar des Völkerbunds benannte Dokument wurde auf der Grundlage eines zwischenstaatlichen Memorandums vom 5. Juli 1922 an Russland-Flüchtlinge ausgestellt, später an weitere Gruppen von de facto Staatenlosen. Es diente als weithin akzeptiertes Identitäts- und Reisedokument. ${ }^{116}$ Letztlich verlassen aber weder der Nansen-Pass noch sein heutiger Nachfolger, der Reiseausweis gemäß der Genfer Flüchtlingskonvention (GFK), das zwischenstaatliche Paradigma ganz. Sie gehen mit der Anomalie um, dass der Staat der Staatsangehörigkeit die ihm im dreipoligen Fremdenrechtsverhältnis zugedachte Rolle, seinen Angehörigen Schutz zu gewähren, nicht einnimmt, ja sogar selbst zur Ursache für die aktuelle Schutzbedürftigkeit des Flüchtlings geworden ist. Der Ausstellerstaat des Reisedokuments rückt gegenüber dem faktisch staatenlos Geworden treuhänderisch in die Stellung ein, die er gegenüber eigenen Staatsangehörigen einnimmt: Er erklärt sich zur Rückübernahme bereit, sofern dem Flüchtling nicht anderweitig dauerhafter Schutz vor Verfolgung gewährt wird.

Der bessere Anwärter für das zentrale Rechtsinstitut der individualrechtlichen Perspektive ist das Prinzip des non-refoulement. Das in Art. 33 Abs. 1 GFK normierte Verbot, einen Flüchtling durch Verweigerung der Einreise oder durch Erzwingung der Ausreise einer Verfolgungssituation auszusetzen, kann als Archetyp einer individualrechtlich fundierten Beschränkung des staatlichen Zulassungs- bzw. Ausweisungsermessens gelten. Zwar ist das flüchtlingsrechtliche Refoulementverbot nicht als individuelles Asylrecht formuliert und sollte diesen Gehalt seiner Entstehungsgeschichte nach auch nicht besitzen. ${ }^{117}$ Das Zurückweisungsverbot verdichtet sich aber zu einem Anspruch auf provisorische Gebietszulassung, wenn nur auf diesem Weg der gebotene Schutz vor Verfolgung - ggf. auch in einem anderen Staat, der zur Aufnahme und Schutzgewähr bereit ist - sichergestellt werden kann. ${ }^{118}$ Neben der flüchtlingsrechtlichen Spezialregelung ergeben sich Refoulementverbote aus weiteren völkervertragsrechtlichen Quellen, insbesondere Art. 3 der UN-Antifolterkonventi-

115 M. Herdegen, Der „Fremde“ im Völkerrecht, in: FS Doehring z. 80. Geb., 2000, S. 13, 14.

116 C. M. Skran, Refugees in Inter-war Europe, 1995, S. 102-122, M. R. Marrus, The Unwanted, 1985 , S. 93-96; J. Oltmer, Flucht, Vertreibung und Asyl im 19. und 20. Jahrhundert, in: IMIS-Beiträge Nr. 20 (2002), S. 107, 127-131.

117 W. Kälin, Das Prinzip des Non-Refoulement, 1982, S. 6-11; E. Reichel, Das staatliche Asylrecht ,im Rahmen des Völkerrechts“, 1987, S. 39-57.

118 K. Hailbronner/J. Gogolin, Aliens, in: R. Wolfrum (Hrsg.), MP-EPIL online (Stand: Mai 2009), Rn. 15; J. C. Hathaway, The Rights of Refugees under International Law, 2005, S. 300-370, insb. S. 315 f.; M. Hong, Asylgrundrecht und Refoulementverbot, 2008, S. 176-178. 
on sowie implizit aus Art. 6, 7 UN-Zivilpakt und Art. 3 EMRK. ${ }^{119}$ Das Verbot der Aus- oder Zurückweisung in Verfolgung wird darüber hinaus von vielen Autoren als eine Norm des Völkergewohnheitsrechts angesehen, ${ }^{120}$ von manchen sogar als ius cogens. ${ }^{121}$ Andere sind mit Blick auf die Unterschiede zwischen den vertraglichen Regelungen und eine fehlende Rechtsüberzeugung der Staaten skeptisch. ${ }^{122}$

Bemerkenswerterweise hat das Prinzip des non-refoulement seine historischen Wurzeln gar nicht im individualrechtlichen Kontext, sondern entwickelte sich aus dem völkerrechtlichen Institut der Asylgewähr, also der Befugnis eines Staates, die Auslieferung politischer Straftäter zu verweigern. ${ }^{123}$ Diesem zwischenstaatlichen Verständnis des Asylrechts entspricht innerstaatlich ein Auslieferungs- oder Ausweisungsverbot, ohne dass dieses notwendig als Individualrecht ausgestaltet sein müsste. Noch Art. 14 der Allgemeinen Erklärung von 1948 ist, trotz seiner subjektiven Formulierung, von diesem zwischenstaatlichen Verständnis des Asylrechts geprägt, wenn seinem „right to seek and to enjoy in other countries asylum from persecution“ bezeichnenderweise keine Schutzgewährverpflichtung desjenigen Staates entspricht, an den das Asylersuchen gerichtet ist. ${ }^{124}$

Der grundlegende Verständniswandel hin zu einem humanitär motivierten Verbot, schutzbedürftige Migranten durch aufenthaltsbeendende Maßnahmen einer Verfolgungssituation auszusetzen, vollzog sich erst nach dem Zweiten Weltkrieg - nicht zuletzt in Ansehung der Massenfluchtbewegungen, die dieser ausgelöst hatte. ${ }^{125}$ In seiner Konsequenz wird der Flüchtlingsschutz heute ganz überwiegend als sekundärer Menschenrechtsschutz verstanden und ist damit von einer individualrechtlichen Perspektive dominiert. ${ }^{126}$ Überdies ist die Zugehörigkeit des Prinzips des non-refoulement zu den grundlegenden Menschenrechten allgemein anerkannt und in den

119 Ausf. K. Wouters, International Legal Standards for the Protection from Refoulement, 2009.

$120 \mathrm{Ph}$. C. W. Chan, The Protection of Refugees and Internally Displaced Persons, Int'l J. of Human Rights 10 (2006), S. 231, 232-235; A. Duffy, Expulsion to Face Torture?, IJRL 20 (2008), S. 373, 383-389.

121 A. Farmer, Non-Refoulement and Jus Cogens, GILJ 23 (2008), S. 1-38; J. Allain, The Jus Cogens Nature of Non-Refoulement, IJRL 13 (2001), S. 533-558.

122 K. Wouters, International Legal Standards for the Protection from Refoulement, 2009, S. 525-530, 577; zurückhaltend auch D. A. Martin, The Authority and Responsibility of States, in: T. A. Aleinikoff/V. Chetail (Hrsg.), Migration and International Legal Norms, 2003, S.31, 37 f., und G. S. Goodwin-Gill, The Refugee in International Law, 2. Aufl. 1996, S. 170 f.; nunmehr aber ders./ J. McAdam, The Refugee in International Law, 3. Aufl. 2007, S. 345-354.

123 O. Kimminich, Der internationale Rechtsstatus des Flüchtlings, 1962, S. 67-79; K.-L. Backsmann, Das Asylrecht in Preußen zur Zeit der Weimarer Republik, 2000, S. 16-23; zur Genese aus vormodernen Formen des Asyls K. Härter, Die Formierung transnationaler Strafrechtsregime, Rg 18 (2011), S. 36, 54-62.

124 E. Reichel, Das staatliche Asylrecht „im Rahmen des Völkerrechts“, 1987, S. 34 f.

125 G. S. Goodwin-Gill, The Refugee in International Law, 2. Aufl. 1996, S. 119-121; O. Kimminich, Der internationale Rechtsstatus des Flüchtlings, 1962, S. 160-176.

126 T. Löhr, Die kinderspezifische Auslegung des völkerrechtlichen Flüchtlingsbegriffs, 2009, S. 61 f., mit zahlr. Nachw. 
Vertragsstaaten der EMRK mit einem effektiven Durchsetzungsmechanismus versehen. In seinem Kerngehalt schützt es davor, durch aufenthaltsrechtliche Maßnahmen einer krass menschenrechtswidrigen Behandlung ausgesetzt zu werden, insbesondere der Folter oder unmenschlicher oder erniedrigender Strafe. ${ }^{127}$

Das Prinzip des non-refoulement besitzt also aufenthaltsrechtliche Regelungsgehalte, die sich je nach Ausgangskonstellation im Rechtsanspruch auf Unterlassen aufenthaltsbeendender Maßnahmen oder auf Duldung der Einreise und Begründung provisorischen Aufenthalts ausdrücken. Mit dieser doppelten Auszeichnung - als Pionier für im Völkerrecht begründete Rechte des Einzelnen und als Beschränkung der staatlichen Befugnis zur Regelung der Einreise und des Aufenthalts von Ausländern bringt das Refoulementverbot gleich zwei Dogmen eines souveränitätszentrierten Völkerrechtsverständnisses ins Wanken. ${ }^{128}$ Es kann damit allemal als ein würdiger Vertreter einer individualrechtlichen Perspektive auf Migration gelten.

\section{Anliegen der Migrationssteuerung: Ermächtigung von Migranten}

Das übergreifende Anliegen, das die individualrechtliche Perspektive in die Steuerung der Migration einbringt, ist vielleicht mit „Ermächtigung von Migranten“ am besten auf den Begriff gebracht. Er ist angelehnt an das Konzept des empowerment, das im Zuge der US-Bürgerrechtsbewegung entstanden ist und heute in einer Vielzahl von Kontexten verwendet wird, von der Managementlehre bis zum Menschenrechtsdiskurs. ${ }^{129}$ Eine Stärkung durch Berechtigung(en) kann an drei Stellen des Aufenthaltsrechtsverhältnisses ansetzen: Ermächtigung von Migranten bei der Aushandlung seines aufenthaltsrechtlichen Status durch Ausstattung mit Verfahrensrechten gegenüber Behörden und Gerichten, sodann Ermächtigung durch Aufwertung des einmal erlangten Status zu einer individuellen Rechtsposition, die gegen willkürlichen Entzug geschützt ist, und schließlich Ermächtigung durch subjektive Rechte auf Begründung oder Fortsetzung eines Aufenthalts, sei es durch Rechtsansprüche auf einen bestimmten Aufenthaltsstatus, sei es durch materielle Rechtspositionen, die im Rahmen aufenthaltsrechtlicher Abwägungsprozesse beachtet werden müssen.

Die individualrechtliche Perspektive tendiert im Grundsatz zu liberalen zuwanderungspolitischen Positionen, denn inhaltlich drängt sie - gewissermaßen umgekehrt zur Gefahrenabwehr-Perspektive - stets auf die Formulierung von positiven Kriterien, welchen Personen eine individualrechtlich geschützte Rechtsposition einzuräumen sei. Der spezifische Zugriff auf Fragen der Migrationssteuerung, der mit der

127 EGMR, Nr. 14038/88, Soering/Vereinigtes Königreich, Ser. A Nr. 161, \$S 88-91; für eine Rekonstruktion des Refoulementverbots aus dem Menschenwürdegehalt der Grundrechte des GG siehe M. Hong, Asylgrundrecht und Refoulementverbot, 2008, S. 153-198.

128 Vgl. O. Kimminich, Der Aufenthalt von Ausländern in der Bundesrepublik Deutschland, 1980, S. 32-37; K. Hailbronner, Ausländerrecht und Verfassung, NJW 1983, S. 2105, 2110 f.

129 Im migrationsrechtlichen Kontext siehe H. Bielefeldt, Menschenrechte in der Einwanderungsgesellschaft, 2007, S. 158. 
Wahrnehmung von Migranten als Rechtssubjekten einhergeht, wäre dann die Steuerungsbegrenzung. In begrifflicher Anleihe bei der Charakterisierung eines liberalen Strafrechts als „Verbrechensbekämpfungsbegrenzungsrecht“, die auf den Frankfurter Juristen Wolfgang Naucke zurückgeht, ${ }^{130}$ könnte man ein liberales, auf Rechten basiertes Migrationsrecht vielleicht insgesamt als ein „Migrationssteuerungsbegrenzungsrecht" bezeichnen. Die Begrenzung des Steuerungsgedankens in der Migrationspolitik wäre dann die wesentliche Leistung der rechtsstaatlichen Verrechtlichung der Migrationssteuerung. Diese Erzählung von der Verrechtlichung der Migrationspolitik als fortschreitende Zivilisierung ursprünglicher Gewaltverhältnisse hätte jedoch - ganz wie ihre Schwester von der fortschreitenden Humanisierung der Strafe - zuerst den Einwand zu entkräften, dass die Verrechtlichung immer auch mit einer Perfektionierung des staatlichen Zugriffs auf das soziale Geschehen verbunden ist. Das emanzipatorische und das disziplinierende Potenzial des Rechts, seine berechtigende Seite und seine "gouvernementale“ Seite als Technik der Macht, bilden eine Melange, die sich gegen historische Simplifizierungen sperrt. ${ }^{131}$

Vorzugswürdig ist deshalb eine Deutung, nach der die allgemeine These vom Doppelauftrag des Öffentlichen Rechts, gleichermaßen der Konstituierung und der Begrenzung hoheitlicher Befugnisse zu dienen, ${ }^{132}$ auch für das Verhältnis von individuellen Berechtigungen für Migranten zum Recht der Migrationssteuerung maßgeblich ist. Demnach begrenzen auf der einen Seite individuelle Rechte von Migranten in der Tat die hoheitlichen Möglichkeiten der Migrationssteuerung, weil bestimmte Handlungsoptionen als rechtswidrig ausgesondert sind. Es ist dieses Verständnis von individuellen Rechten, das der Beobachtung eines „liberalen Paradoxes“ der Migrationssteuerung zugrunde liegt. ${ }^{133}$ Auf der anderen Seite erfüllen individuelle Rechte nicht bloß eine negative, abwehrrechtliche Funktion, sondern sie können positives Handeln verlangen und die hoheitliche Steuerung inhaltlich ausrichten. Die Möglichkeit für die Einzelnen, von individuellen Rechten effektiv Gebrauch zu machen, wird hier als ein Steuerungsziel gesetzt. Der Schutz individueller Rechte und die Sicherung ihrer Ausübungsvoraussetzungen können als (auch) im öffentlichen Interesse liegend verstanden werden. ${ }^{134}$ Grundrechtstheoretisch setzt dies bei Grundrechtsgehalten an, die einer legislativen Ausgestaltung bedürfen bzw. deren Wirk-

130 W. Naucke, Die Kriminalpolitik des Marburger Programms 1882, ZStW 94 (1982), S. 525, 564.

131 Siehe nur M. Foucault, Überwachen und Strafen, 1991; F. L. Neumann, Der Funktionswandel des Gesetzes im Recht der bürgerlichen Gesellschaft, Zeitschrift für Sozialforschung 6 (1937), S. 542, $569 \mathrm{f}$. und $594 \mathrm{f}$.

132 E. Schmidt-Aßmann, Das allgemeine Verwaltungsrecht als Ordnungsidee, 2006, 1/30-32, passim.

133 Zu diesem Theorem J. F. Hollifield, Immigrants, Markets, and States, 1992, S. 222-232.

134 Zur Verknüpfung individueller und öffentlicher Interessen im Bereich der Grundrechte siehe R. Uerpmann, Das öffentliche Interesse, 1999, S. 61-69, 132; zur abw. ausländerrechtlichen Terminologie ebd. 46. 
samkeit durch gesetzgeberische Aktivität verstärkt wird. ${ }^{135}$ Menschenrechtsdogmatisch formuliert geht es um positive Verpflichtungen ${ }^{136}$ bzw. um eine „obligation to fulfil“ “. ${ }^{137}$ Rechtsphilosophisch kann man Migrationssteuerung mittels aufenthaltsrechtlicher Gesetzgebung als „demokratische Iterationen“ universeller Menschenrechte deuten und so das Gestaltungspotenzial anerkennen, das dem Prozess der „wiederholenden“ Implementation kosmopolitischer Normen inhärent ist. ${ }^{138}$

Aus einer individualrechtlichen Perspektive auf das Migrationsgeschehen kann sich der Zusammenhang von Steuerung und Rechten noch in einer weiteren Hinsicht darstellen, nämlich in der Nutzung von individuellen Rechten als Steuerungsinstrument. Dies meint ein Verhältnis von zentraler Gesetzgebung und dezentralem Vollzug, das als funktionale Subjektivierung bezeichnet werden kann. ${ }^{139}$ Der Grundgedanke ist, dass die Schaffung von subjektiven Rechten (zumindest auch) dazu dient, legislativen Steuerungsanliegen zur effektiveren Durchsetzung zu verhelfen, indem die Verwaltung auf ein bestimmtes Handeln festgelegt wird und die interessierten Bürger dieses mit Hilfe der Gerichte einklagen können. Im AufenthG wird die Technik der Programmierung der Verwaltung durch Rechtsansprüche auf Erteilung eines Aufenthaltstitels an diversen Stellen eingesetzt. Besonders bedeutsam ist der Hebel der funktionalen Subjektivierung im Verhältnis von EU-Gesetzgeber und mitgliedstaatlichen Vollzugsbehörden, wo hierarchische Steuerungsressourcen wie die Programmierung der Verwaltung durch Verwaltungsvorschriften der politischen Spitze fehlen. Entsprechend zeichnet sich ab, dass der Unionsgesetzgeber individuelle Rechtsansprüche als migrationsrechtliche Steuerungsressource auch dort einsetzt, wo dies im staatlichen Kontext unüblich ist, etwa bei der sog. Blue Card für qualifizierte Erwerbsmigranten. ${ }^{140}$ Dies spricht im Übrigen dafür, die individualrechtliche Perspektive nicht auf einen menschenrechtlichen Ansatz engzuführen, da die für Steuerungszwecke eingesetzten Rechte nicht notwendig diese herausgehobene Qualität besitzen müssen.

135 Siehe M. Cornils, Die Ausgestaltung der Grundrechte, 2005, S. 2-31; für grundrechtsrelevante Statusentscheidungen ist ein besonderer Bedarf nach organisations- und verfahrensrechtlicher Ausgestaltung anerkannt, siehe E. Denninger, Staatliche Hilfe zur Grundrechtsausübung durch Verfahren, Organisation und Finanzierung, in: J. Isensee/P. Kirchhof (Hrsg.), HStR V, 2. Aufl. 2000, $\mathbb{S} 113$ Rn. 15 f.

136 C. Dröge, Positive Verpflichtungen der Staaten in der Europäischen Menschenrechtskonvention, 2003, S. 87-100.

137 M. Sepúlveda, The Nature of the Obligations under the International Covenant on Economic, Social and Cultural Rights, 2003, S. 239-241; J. Künzli, Zwischen Rigidität und Flexibilität, 2001, S. 210213, 279-283.

138 Zur Idee der demokratischen Iteration siehe S. Benhabib, Die Rechte der Anderen, 2009, S. 168-205; dies., Democratic Iterations, in: R. Post (Hrsg.), Another Cosmopolitanism, 2006, S. 45, 47-50.

139 Im europarechtlichen Kontext vgl. M. Ruffert, Subjektive Rechte im Umweltrecht der Europäischen Gemeinschaft, 1996; J. Masing, Die Mobilisierung des Bürgers für die Durchsetzung des Rechts, 1996.

140 Zweifelnd D. Thym, Migrationsverwaltungsrecht, 2010, S. 151. 


\section{G. Kopplung der Perspektiven als Aufgabe des Migrationsrechts}

Der abschließende Teil entwickelt einige Überlegungen dazu, wie das Recht mit dem Befund der Perspektivenpluralität umgehen kann. Die Frage ist, welchen Beitrag das Migrationsrecht zur Zusammenführung der Perspektiven und zur Vorrangentscheidung im Fall der Kollision leisten kann. Beide Aspekte, Bündelung und Konfliktregulierung, sollen im Folgenden unter dem Begriff „Kopplung“ zusammengefasst werden. Die hier vertretene These lautet, dass das Recht zwei zentrale Aufgaben bei der Kopplung der unterschiedlichen Perspektiven auf das Migrationsgeschehen erfüllen kann: erstens die strukturelle Sicherung von Multiperspektivität als einem Mechanismus der Rationalitätsgewährleistung für hoheitliches Handeln und zweitens die gezielte Priorisierung und relative Gewichtung von Perspektiven als eine Steuerungsressource.

Die erste Aufgabe, Sicherung von Multiperspektivität, stellt sich vor allem in Situationen, in denen die mit den einzelnen Perspektiven assoziierten Organisationseinheiten stark verselbständigt sind und es an institutionalisiertem Zwang zur Perspektivenkopplung fehlt. Die Hintergrundannahme ist die, dass alle fünf Perspektiven der Migrationssteuerung - jedenfalls in der wertungsoffenen Form, in der sie hier eingeführt wurden - Gemeinwohlanliegen zur Sprache bringen, die bei einem migrationspolitischen Gesamtansatz nicht ausgeblendet werden sollten. Diese problematische Situation besteht bei den internationalen Organisationen, die an einer globalen Governance der Migration interessiert sind. ${ }^{141}$ Für den staatlichen und unionsrechtlichen Kontext stellt sich das Fragmentierungsproblem weniger scharf. Beide Handlungsebenen verfügen über institutionelle Mechanismen der Herstellung von Multiperspektivität, insbesondere über grundsätzlich ergebnisoffene Rechtsetzungsverfahren in zentralen Gremien, die nicht per se auf eine bestimmte Perspektive festgelegt sind und somit als prozedurale Gewährleistung von Multiperspektivität verstanden werden können.

Im Zentrum des Migrationsrechts steht der zweite Aspekt, die Nutzung der Perspektivenpluralität als Steuerungsressource. Hier steht der konflikthafte Charakter der unterschiedlichen Verständnisse von Migration im Vordergrund; die Bezeichnung als „konkurrierende Perspektiven“ zeigt diesen Wettbewerb bewusst an. Dieses Ringen um das relative Gewicht der Perspektiven ist ein legitimer politischer Vorgang, der nicht durch juristische Interpretations- und Abwägungsprozesse substituiert werden kann, zumal die Perspektiven selbst erst inhaltlich gefüllt werden müssen, um zu einem abwägungsfähigen Belang zu werden. Multiperspektivität hat einen Eigenwert als rationale Methode des Suchens nach balancierten Ergebnissen, sie darf aber nicht zu einem ungeschriebenen Gebot überhöht werden, dass alle Perspektiven in allen mokratie in der Weltgesellschaft, 2009, S. 185-193. 
Konstellationen möglichst gleichmäßig berücksichtigt werden müssten. Vielmehr liegt ein wesentlicher Hebel für die rechtliche Steuerung der Migration darin, das in der Perspektivenpluralität liegende Potenzial zur relativen Gewichtung von Anliegen zu nutzen, indem je nach Konstellation eine Perspektive gegenüber anderen Priorität genießt, wohingegen sie in anderen Konstellationen gezielt ausgeblendet wird.

Dieser Aufgabe hat sich das Migrationsrecht auf allen Stufen der Normenhierarchie zu stellen. Verfassungs- und völkerrechtliche Vorgaben in Gestalt von Grundrechten bzw. Menschenrechten strukturieren die legislativen Perspektivenkopplungen in gewissem Umfang vor und verengen die administrativen Handlungskorridore. Diese Vorgaben leisten eine erste horizontale Differenzierung in aufenthaltsrechtliche Kategorien, bei denen die Perspektiven je unterschiedlich gewichtet sind. Den Gesetzgebern obliegt die Übersetzung in administrativ abzuarbeitende Arbeits- und Entscheidungsprogramme. Hier ist der Ort politisch zu verantwortender Gestaltung, indem eine legislative Ausdifferenzierung der Rechtsregimes für unterschiedliche Aufenthaltszwecke stattfindet und Einwanderungschancen eröffnet oder verschlossen werden. Häufig aber findet die maßgebliche Bündelung der Perspektiven erst auf der Ebene der individuellen Verwaltungsentscheidung statt. Die dabei geforderte Güter- und Interessenabwägung im Rahmen von Ermessensentscheidungen kann als spezifisch verwaltungsrechtliche Form der Perspektivenkopplung verstanden werden. Die Perspektivenpluralität und das Erfordernis, diese im Medium des Rechts zu bewältigen, finden schließlich auch Ausdruck in komplexen interadministrative Verfahrensarrangements, bei denen unterschiedliche Fachbehörden in die Entscheidungsfindung einbezogen sind.

Aus steuerungstheoretischer Sicht liegt es deshalb nahe, die Aufenthaltsgenehmigung als den zentralen Knotenpunkt der Perspektivenkopplung im Aufenthaltsrecht zu verstehen. Die Festlegung der materiellen und verfahrensmäßigen Voraussetzungen der förmlichen Genehmigung des Aufenthalts sowie der Inhalt des damit zugewiesenen aufenthaltsrechtlichen Status sind der maßgebliche verwaltungsrechtliche Ort, an dem die Bündelung und relative Gewichtung der konkurrierenden Perspektiven der Migrationssteuerung stattfindet. Dies bedeutet zugleich, das Institut der Aufenthaltsgenehmigung aus einer traditionell starken Bindung an die Perspektive der Gefahrenabwehr herauszulösen, für die der aufenthaltsrechtliche Erlaubnisvorbehalt vor allem der präventiv-polizeilichen Kontrolle der Bedrohungspotenziale des Ausländeraufenthalts dient. In der Folge dieses grundlegenden Verständniswandels ist das Aufenthaltsrecht insgesamt vom Institut der Aufenthaltsgenehmigung her zu bauen, nicht von der Ausweisung: Die Regelungen über die Begründung und stufenweise Verfestigung des aufenthaltsrechtlichen Status, nicht das Regime der aufent- 
haltsbeendenden Maßnahmen sind die entscheidenden Instrumente, mit denen die Konflikte bewältigt werden müssen, die das Aufenthaltsrecht beherrschen. ${ }^{142}$ halts, in: K. Barwig u.a. (Hrsg.), Ausweisung im demokratischen Rechtsstaat, 1996, S. 23, 24 und 38. 